\title{
Neurons in Macaque Inferior Temporal Cortex Show No Surprise Response to Deviants in Visual Oddball Sequences
}

\author{
Dzmitry A. Kaliukhovich and Rufin Vogels \\ Laboratorium voor Neuro-en Psychofysiologie, KU Leuven Medical School, Leuven, BE-3000, Belgium
}

\begin{abstract}
Many studies measured neural responses in oddball paradigms, showing a different response to the same stimulus when presented with a low (deviant) compared with a high probability (standard) in a sequence. Such a differential response is manifested in event-related potential studies as the mismatch negativity (MMN) and has been observed in several sensory modalities, including vision. Other studies showed that stimulus repetition suppresses the neural response. It has been suggested that this adaptation effect underlies the smaller responses to the standard compared with the deviant stimulus in oddball sequences. However, the MMN may also reflect the violation of a prediction based on the sequence of standards, i.e., a surprise response. We examined the presence of a surprise response to deviants in visual oddball sequences in macaque (Macaca mulatta) inferior temporal (IT) cortex, a higher-order cortical area. In agreement with visual MMN studies, single-unit IT responses were greater for the deviant than for the standard stimuli. However, single IT neurons showed no greater response to the deviant stimulus in the oddball sequence than to the same stimulus presented with the same probability in a sequence that consisted of many stimuli. LFPs also showed no evidence of a surprise response. These data suggest that stimulus-specific adaptation, without a surprise-related boost of activity to the deviant, underlies the responses in visual oddball sequences even in higher visual cortex. Furthermore, we show that for IT neurons such adaptive mechanisms take into account a relatively short stimulus history, with weaker effects at longer time scales.
\end{abstract}

Key words: adaptation; inferior temporal cortex; macaque; mismatch negativity; repetition suppression; surprise response

\section{Introduction}

Over the last few decades a large number of studies have measured neural responses in oddball paradigms where a stimulus, the standard, was presented with a high probability while another stimulus, the deviant, was presented unexpectedly with a low probability. These studies demonstrated a different neural response to the deviant compared with the standard, which in event-related potentials (ERPs) manifested as the mismatch negativity (MMN). Although first demonstrated in the auditory domain (Näätänen et al., 1978), a MMN is also present in visual (Czigler et al., 2002) and somatosensory modalities (Kekoni et al., 1997). Understanding the mechanisms underlying these responses in oddball paradigms is important because these paradigms are used in studies of neurological and psychiatric diseases (Duncan et al., 2009).

In all sensory modalities, stimulus repetition usually produces a suppression of the neural response. Such adaptation effects or

Received May 28, 2014; revised July 18, 2014; accepted July 28, 2014.

Author contributions: D.A.K. and R.V. designed research; D.A.K. performed research; D.A.K. analyzed data; D.A.K. and R.V. wrote the paper.

This work was supported by Fonds voor Wetenschappelijk Onderzoek Vlaanderen (G.0582.12N), Geconcerteerde Onderzoeksactie (GOA/10/019), Interuniversitaire Attractiepool and Programma Financiering (PF 10/008). We thank P. Kayenbergh, G. Meulemans, I. Puttemans, M. De Paep, W. Depuydt, and S. Verstraeten for technical assistance, and Dr J. Taubert, Dr H. Op de Beeck, and K. Vinken for critical reading of a draft of this paper.

The authors declare no competing financial interests.

Correspondence should be addressed to Rufin Vogels, Laboratorium voor Neuro- en Psychofysiologie, KU Leuven Medical School, Campus Gasthuisberg, 0\&N2, Herestraat 49, bus 1021, Leuven, BE-3000, Belgium. E-mail: Rufin.Vogels@med.kuleuven.be.

DOI:10.1523/JNEUROSCI.2154-14.2014

Copyright $\odot 2014$ the authors $\quad 0270-6474 / 14 / 3412801-15 \$ 15.00 / 0$ repetition suppression may account for the differential responses to the standard compared with deviant stimulus in an oddball paradigm, because adaptation is largely stimulus-specific. However, ERP studies appear to demonstrate that the MMN also reflects the violation of a prediction based on the sequence of standards (Näätänen et al., 2005; Garrido et al., 2009; Kimura, 2012). A common strategy to disentangle the "surprise" response (Barto et al., 2013) from stimulus-specific adaptation is to compare the response for the deviant in the oddball sequence to that for the same stimulus, presented with the same probability, but in a sequence that consists of many stimuli (an "equiprobable" sequence). With this paradigm, ERP studies reported surpriserelated responses in both auditory (Jacobsen and Schröger, 2001) and visual modalities (Czigler et al., 2002). However, multiunit and local field potential (LFP) studies in rats (Farley et al., 2010) and monkeys (Fishman and Steinschneider, 2012) failed to find a surprise response in the primary auditory cortex. Yet, it may still be present in higher auditory areas (Szycik et al., 2013).

Stimulus-specific adaptation is ubiquitously present in macaque inferior temporal (IT) cortex (Gross et al., 1967; Miller et al., 1991a,b; Sawamura et al., 2006; Liu et al., 2009; Kaliukhovich and Vogels, 2012), an area involved in the coding of visual object properties (Logothetis and Sheinberg, 1996; Tanaka, 1996; DiCarlo et al., 2012). Given its high level in the visual hierarchy and the finding of prediction-related responses in this cortex (Meyer and Olson, 2011), IT is an area likely to exhibit surprise responses to deviants in oddball sequences. Here, we examine single-unit responses and LFPs in macaque IT to standards and deviants in 
oddball sequences and compare these responses to those for identical stimuli in an equiprobable sequence. Because ERP studies demonstrated a visual MMN when subjects were not attending to the stimuli (Czigler, 2007), the monkeys were performing a passive fixation task during the recordings. Anticipating the results, we show that the single-unit IT responses in visual oddball sequences merely reflect stimulus-specific adaptation. Examination of the responses in these sequences allowed us to specify the time course of the stimulus-specific adaptation in this area.

\section{Materials and Methods}

\section{Subjects}

Two rhesus macaques (Macaca mulatta; male Monkey $\mathrm{P}$ and female Monkey K, weighing 8.5 and $6.8 \mathrm{~kg}$, respectively, both left hemisphere) served as subjects. Animal care and experimental procedures met the national and European guidelines and were approved by the Ethical Committee for Animal Experiments of the KU Leuven.

Details about implants and surgery can be found in De Baene and Vogels (2010) and Kaliukhovich and Vogels (2011). The localization of the recording chamber was guided and verified by magnetic resonance imaging (MRI) scans obtained prior and during the course of recording sessions. Estimations of the recording positions were obtained by the visualization of glass capillaries filled with the MRI opaque copper sulfate $\left(\mathrm{CuSO}_{4}\right)$ inserted into the recording chamber grid (Crist Instruments) at predetermined positions. Recording positions were estimated based on the MRI visualization of these markers, the microdrive depth readings of the white/gray matter transitions relative to the grid base and of the skull.

Recordings were performed in the lower bank of the superior temporal sulcus and the lateral convexity of the IT cortex. The anterior-posterior coordinates of the recording positions ranged between 18 and $21 \mathrm{~mm}$, and 16 and $18 \mathrm{~mm}$ anterior to the auditory meatus in Monkeys $\mathrm{P}$ and $\mathrm{K}$, respectively, whereas the corresponding medial-lateral coordinates in those monkeys ranged between 20 and $23 \mathrm{~mm}$ lateral to the midline.

\section{Recordings}

Apparatus and recording procedures were identical to those of De Baene and Vogels (2010) and Kaliukhovich and Vogels (2011). LFPs and spikes were recorded simultaneously from the same microelectrode using a Plexon data acquisition system. The recordings were performed with epoxy-coated tungsten microelectrodes (FHC; $\sim 1 \mathrm{M} \Omega$ impedance in situ) lowered with a Narishige microdrive through a guide tube that was fixed in a Crist grid. The grounded guide tube served as the reference. Recorded signals were preamplified with a headstage having an input impedance of $>1 \mathrm{G} \Omega$. The signals were split into spiking activity (bandpassed signal between $250 \mathrm{~Hz}$ and $8 \mathrm{kHz}$ ) and LFPs (band-passed signal between 0.7 and $170 \mathrm{~Hz}$ sampled at $1 \mathrm{kHz}$ ). Spiking activity of isolated single neurons was thresholded online, and spike waveforms were saved at $40 \mathrm{kHz}$. In addition, we used Offline Sorter (Plexon) to verify the single-cell isolation.

Eye position was measured online with an infrared-based eye tracking system (ISCAN EC-240A; $120 \mathrm{~Hz}$ sampling rate). The analog eye movement signal was saved with a sampling frequency of $1 \mathrm{kHz}$. Stimulus and behavioral events were stored for later offline analysis on a computer that was synchronized with the Plexon data acquisition system.

\section{Stimuli}

We used two sets of stimuli, each consisting of 52 images of objects belonging to different categories. The first stimulus set was identical to that used in the study by Kaliukhovich and Vogels (2012) and included color images of human and monkey faces, human and monkey bodies, mammals, birds, fishes, snakes, insects, trees, fruits, fractals, and manmade objects. Similar to the first stimulus set, the second stimulus set comprised 32 grayscale images of the real-world objects, such as, human and monkey bodies, mammals, birds, fruits, sculptures, and manmade objects. These stimuli were randomly selected from a broader stimulus set used in the fMRI study by Popivanov et al. (2012) (see their Fig. 1). In addition, the second stimulus set included artificially generated grayscale images of five male and five female faces of different identities of approx- imately the same age and ethnicity with each face identity being represented by both the frontal and profile views ( 2 views $\times 5$ male identities +2 views $\times 5$ female identities $=20$ stimuli). The face stimuli were generated by means of FaceGen software (Singular Inversions; http://www.facegen.com). We equated the mean grayscale level across all stimuli of the second stimulus set. The size of the stimuli (maximum of horizontal and vertical dimensions of the bounding box) in both stimulus sets was equated and measured $\sim 5^{\circ}$ of visual angle. The stimuli were shown on a uniform gray background with their centers of mass positioned in the center of a CRT monitor (Phillips Brilliance 202P4, frame rate $=60 \mathrm{~Hz}$, resolution $=1024 \times 768$ pixels) located $61 \mathrm{~cm}$ from the subject's eyes. Note that the monkeys were familiar with all stimuli because these were used to train them on a daily basis before the recordings took place. For the sake of clarity, we further refer to the stimuli from the first stimulus set as "natural stimuli" and from the second set as "face stimuli."

In addition, we created 100 scrambled stimuli which were only shown when switching among the conditions in the visual oddball test (Fig. 1). These were generated by selecting 20 color images (size $=1024 \times 768$ pixels) that depict natural scenes and splitting these into squares of equal size $(32 \times 32$ pixels $)$ which were then randomly scrambled to generate a new stimulus. We applied this procedure five times to each of the 20 original images $(5$ times $\times 20$ images $=100$ stimuli). Note that when presented, the scrambled stimuli filled the entire display.

\section{Experimental design and tests}

We used two tests, the search test and the visual oddball test. At the beginning of each recording session, we always launched the search test first when advancing the microelectrode in the targeted area for recordings. The search test was also used to search for responsive single neurons to test them in the visual oddball paradigm. The search test was restarted every time after we collected the desired amount of data in the visual oddball test or after we lost a recorded neuron.

Search test. The search test was identical to that used in the studies of Kaliukhovich and Vogels $(2011,2012)$ and will only be briefly summarized here. The monkeys were required to fixate a red target square $\left(\right.$ size $\left.=0.17^{\circ}\right)$ presented in the center of the monitor. The target appeared at the beginning of each trial and remained visible throughout it unless the monkeys broke fixation. After $300 \mathrm{~ms}$ of stable fixation, the monkeys were foveally presented a stimulus for another $300 \mathrm{~ms}$. Continuous fixation for $300 \mathrm{~ms}$ poststimulus was rewarded by a drop of apple juice and terminated the trial. All 52 stimuli of a selected stimulus set were repeatedly shown in a pseudorandom order. Based on the spiking responses to the presented stimuli, we chose two stimuli (A and B) of which at least one drove the neuron well, and eight nonoptimal stimuli, which evoked little or no response. The 10 selected stimuli were further used in the visual oddball test.

Visual oddball test. Sequences of stimuli were presented to the monkeys with specific stimulus presentation probabilities under three experimental conditions. The stimuli were presented in blocks of 100 presentations, except in a control experiment in which the blocks consisted of 300 presentations. Each experimental condition included several blocks, except for the control experiment in which only one block was presented per condition. Two consecutive blocks of stimuli were always taken from different experimental conditions. In the first condition, two stimuli, A and B, were randomly interleaved and presented to the monkeys with probabilities of 0.9 and 0.1 , respectively (Fig. 1). In the second condition, these probabilities were reversed; that is, stimuli A and $B$ were presented with probabilities of 0.1 and 0.9 , respectively. Thus, each of the two stimuli (A and B), depending on the condition, was presented either frequently (further referred to as a standard) or rarely (further referred to as a deviant) in the oddball sequences. In the third condition, the two stimuli (A and B) were randomly interleaved with eight other ineffective stimuli, and all 10 stimuli were presented equiprobable; that is, with a stimulus presentation probability of 0.1 . The two probe-stimuli, A and B, when presented in this equiprobable condition, are hereby referred to as a reference. Importantly, when using the second stimulus set, the two stimuli A and B used in the oddball sequences were the frontal and profile views of the same face identity, 


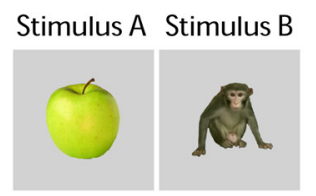

Oddball sequence: $90 \%$ A (Standard) and 10\% B (Deviant)

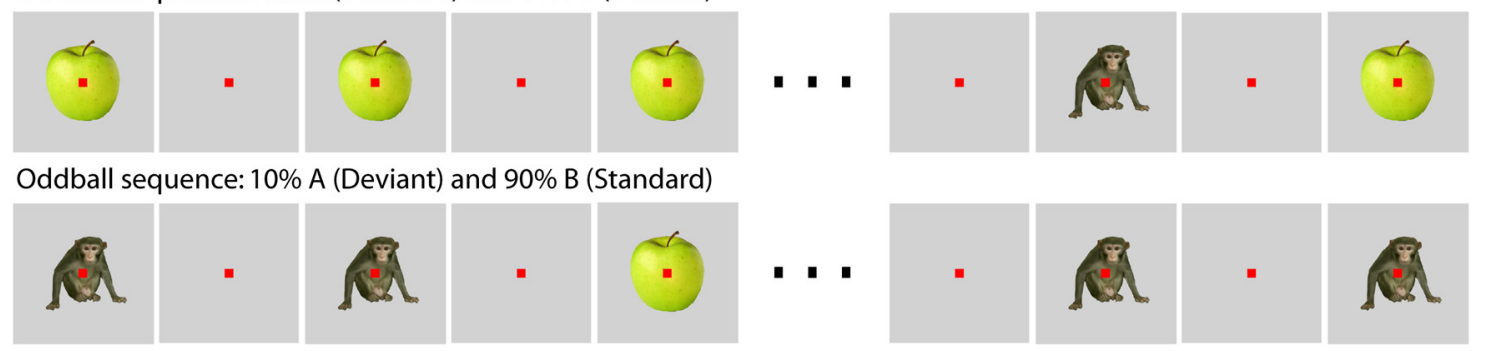

Equiprobable presentation: 10\% A (Reference) and 10\% B (Reference) and 10\% x 8 other stimuli

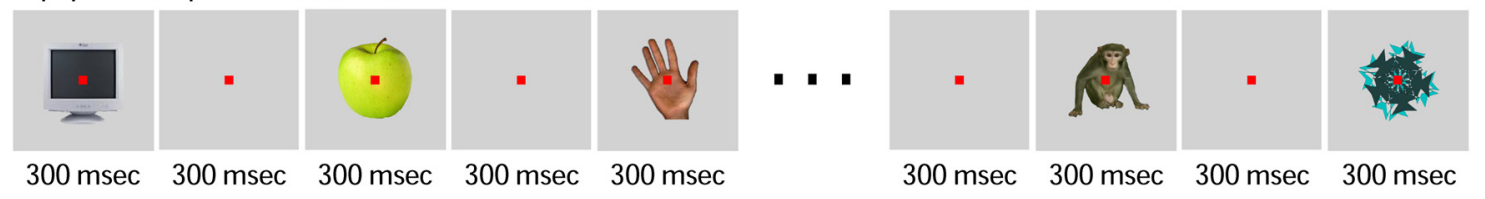

Figure 1. Visual oddball test. Two selected stimuli (A and B) were presented randomly interleaved in blocks of 100 or 300 presentations, with each stimulus being presented either frequently (probability 0.90 , standard) or rarely (probability 0.10 , deviant) within a block (oddball sequences). In addition, the two stimuli $A$ and B were also presented with the same probability (reference), being randomly interleaved with eight weakly effective stimuli in the equiprobable sequence. When fixating on a red target square (here shown not to scale), monkeys were continuously presented a sequence of stimuli, with each stimulus being presented for $300 \mathrm{~ms}$ and followed by a 300-ms-long blank screen. The stimuli A and B differed between neurons and were selected based on responses in a search test.

whereas the remaining eight stimuli used in the third experimental condition were always nonface stimuli.

Like in the search test, monkeys initiated stimulus presentations by passively fixating a small red square $\left(\right.$ size $\left.=0.17^{\circ}\right)$ presented in the center of the monitor for $300 \mathrm{~ms}$. While maintaining their fixation on the fixation target (maximum size of the fixation window: $2.33^{\circ}$ across both monkeys for the natural stimuli and $2.02^{\circ}$ for Monkey $\mathrm{P}$ when using the face stimuli), monkeys were continuously presented with the sequence of stimuli of a block. Each stimulus was shown for $300 \mathrm{~ms}$ and was followed by a 300 -ms-long blank screen (Fig. 1). Stimulus presentation was interrupted when either the monkeys broke fixation or all stimuli of the current block were presented. Stimulus presentations during which the monkeys broke fixation, i.e., a fixation break that occurred during the 300-ms-long stimulus presentation with at least one frame of the stimulus being presented, contributed to the overall statistics of stimulus presentations within that block. Note that the fixation breaks during the stimulus presentations occurred rather infrequently: the mean percentage of stimulus presentations that were aborted per block (across both monkeys and stimulus sets) was 5.5\% (range across the three stimulus conditions: $0.7 \%$ ). While fixating, monkeys were delivered a drop of apple juice. The juice was delivered using a reinforcement schedule in which the longer the monkey fixated the faster and the more frequently the animal received juice. Juice was delivered in-between two consecutive stimulus presentations, and importantly, its delivery was not linked to a particular stimulus.

Except for the first 10 recorded and analyzed neurons/sites in Monkey $P$ when using the natural stimuli, each new block of stimuli in a recording session was preceded by a presentation of 50 randomly chosen scrambled stimuli, with each being serially flashed for $33 \mathrm{~ms}$. During the presentation of these stimuli, no fixation target was shown and monkeys were allowed to make saccades. The time interval between the onset of the first stimulus presentation of a following block and the offset (or a fixation break in the case of aborts) of the last stimulus presentation of a preceding block varied across blocks as it depended on the oculomotor behavior of the animals. For the main experiment (blocks of 100 presentations each), the medians of these intervals ranged from 2106 to $6637 \mathrm{~ms}$ (with minima ranging from 568 to $1521 \mathrm{~ms}$ ) for the first 10 analyzed neurons in Monkey P, and from 7991 to 11752 ms (minima from 7606 to $8793 \mathrm{~ms}$ ) for the remaining neurons across both monkeys and stimulus sets. Be- cause the first 10 recorded neurons in Monkey P showed results similar to those obtained with the natural stimuli in neurons when using the scrambled stimuli in-between the blocks, we pooled here the data across all the neurons of the main test.

The order of conditions was counter-balanced across recorded neurons/sites for each monkey and stimulus set. This counterbalancing was achieved by means of a Latin Square design for three consecutive triplets of blocks (e.g., A-C, B, C, A, C, A, B), with the assignment of condition to block (A, B, or C) being randomized across groups of three triplets. For the data analysis, we equated for each recorded neuron/site the number of completely presented blocks per condition (across both monkeys and stimulus sets: mean $=7.16$ blocks per condition; range, $4-12$ blocks per condition). For the control experiment in which blocks consisted of 300 stimulus presentations, the order of the three conditions was fully counterbalanced across the 30 tested neurons.

\section{Data analysis}

Spiking activity. We only analyzed stimulus presentations for which the monkey maintained fixation from $300 \mathrm{~ms}$ before stimulus onset until 300 ms poststimulus offset. For each thus defined unaborted stimulus presentation, we computed the response to that stimulus using a 300 -mslong analysis window, which started at $50 \mathrm{~ms}$ after stimulus onset. In addition, the baseline firing rate was computed within a window from -50 to $50 \mathrm{~ms}$. To avoid a selection bias, we included all stimulus $\times$ neuron combinations in which the stimulus evoked a significant excitatory response in at least one of three conditions; that is, when presented either as a standard, a deviant, or a reference. A stimulus was considered to evoke a significant response in a particular condition if the mean firing rate to that stimulus, averaged across all unaborted presentations and regardless of the presentation order within the blocks of the selected condition, significantly exceeded the mean baseline firing rate (one-sided Wilcoxon matched-pairs test, Bonferroni corrected for multiple comparisons (three conditions), $p<0.017)$. Note that in most analyses, we used gross responses; that is, no baseline correction was performed. Due to differences in stimulus presentation probability across stimulus conditions, the number of unaborted stimulus presentations $(N)$ varied among conditions (data for the main test: minimum/mean/maximum per stimulus $\times$ neuron combination across both monkeys and stimulus 
sets: $N($ standard $)=268 / 567 / 1032, N($ deviant $)=26 / 63 / 116, N($ reference) $=31 / 64 / 114)$.

To quantify the magnitude of differences in response to the same stimulus among the conditions, for each analyzed stimulus $\times$ neuron combination we computed two contrast indices (Ulanovsky et al., 2004, 2003) defined as follows:

$$
\begin{gathered}
D \_S=(\text { Deviant }- \text { Standard }) /(\text { Deviant }+ \text { Standard }), \\
D \_R=(\text { Deviant }- \text { Reference }) /(\text { Deviant }+ \text { Reference }),
\end{gathered}
$$

with Standard, Deviant, and Reference being the responses to a stimulus presented as a standard, a deviant or a reference, respectively. The $D \_S$ and $D \_R$ values can range from -1 to +1 .

In addition, for each neuron we assessed the relative effectiveness of the two probe-stimuli ( $\mathrm{A}$ and $\mathrm{B}$ ) used in the oddball sequences using the following index:

$$
B \_W=(\text { Best }- \text { Worst }) /(\mid \text { Best }|+| \text { Worst } \mid),
$$

with Best and Worst being the maximum and minimum net responses, respectively, to stimuli $\mathrm{A}$ and $\mathrm{B}$ when those were presented in the equiprobable condition.

Time course of the response to a stimulus within a block. We computed the time course of the population response to a stimulus within a block of stimulus presentations for each condition separately. To compute the time course of the response for each condition and stimulus $\times$ neuron combination, we first averaged the responses to a stimulus across all unaborted presentations of that stimulus at a particular presentation order within the block, and this was done for each stimulus condition, separately. To compute the population time course of the response, we then averaged the derived time courses of the response for each stimulus condition across all analyzed stimulus $\times$ neuron combinations.

To quantitatively assess the differences between the time courses of the population response among the three stimulus conditions, we fitted each of the time courses with a polynomial inverse first order function (Antunes et al., 2010) using nonlinear least-square regression:

$$
r(x)=a+b / x
$$

with $r$ being the mean response to a stimulus at presentation order $x, a$, and $b$ being the free parameters which characterize the asymptote and decay size of the fitted time course, respectively. The statistical significance of the difference between the values of the free parameters among stimulus conditions was tested using bootstrapping. We drew 1000 samples with replacement from the pool of time courses of all analyzed stimulus $X$ neuron combinations, with the size of each sample being equal to the total number of analyzed stimulus $\times$ neuron combinations. For each bootstrap data sample and stimulus condition, we computed the population average time course of the response which we then fitted 100 times using the MATLAB function "lsqnonlin" (default parameters; "trust region reflective" algorithm) of the MATLAB Optimization toolbox. The medians of the derived 100 values served as the estimate for each free parameter of that sample and condition. For each bootstrap data sample, we then computed the difference between the estimated parameter values for each of the three possible pairs of stimulus conditions. The distribution of the 1000 pairwise differences in parameter values was used to define $99 \%$ confidence intervals (percentile method; Efron, 1979) of the difference in parameter values for each free parameter and a pair of stimulus conditions. The difference between the values of a free parameter for a pair of conditions was deemed significant if 0 was excluded from the corresponding confidence interval derived with bootstrapping.

Stimulus history tree analysis. We used a similar analysis of the effect of stimulus history as Ulanovsky et al. (2004). First, for each analyzed stimulus $X$ neuron combination we normalized the responses in the two oddball conditions by dividing the response to each stimulus presentation by the mean response to the same stimulus when presented as a reference in the equiprobable condition. For each unaborted sequence of stimulus presentations, we then selected the responses to stimulus A (A; first order) regardless of the preceding stimulus, to A when following A (AA; second order sequence), to A when following B (BA), to A when following a repetition of A, i.e., the doubled AA (AAA; 3th order sequence), to $A$ when following the doublet $A B(A B A)$, to $A$ when following the doublet $\mathrm{BB}$ (BBA), to A when following the doublet BA (BAA), to A when following the triplet AAA (AAAA; fourth order sequence order) etc. The same was done for stimulus $B$ as a function of the preceding local stimulus sequence ( $\mathrm{B}, \mathrm{BB}, \mathrm{AB}, \mathrm{BBB}, \mathrm{BAB}$, etc.). Note that for this analysis, no fixation aborts were allowed in between the stimulus presentations and the maximum sequence order was 4 . The analysis was performed separately for each stimulus presentation probability. Specifically, the responses to either stimulus were pooled together based on the presentation probability of the selected stimulus in the two oddball conditions, that is, whether that stimulus was presented either as a standard or as a deviant. For each sequence order, the normalized responses were pooled across analyzed stimulus $\times$ neuron combinations and then averaged as a function of the preceding short stimulus sequence. Confidence intervals (95\%) of the mean normalized responses were derived with bootstrapping (1000 bootstrap data samples with replacement; percentile method).

LFPs. As for the analyses of spiking activity, all LFP analyses were based only on unaborted stimulus presentations using the same definition as for the spiking activity. In addition, all unaborted stimulus presentations in which the LFP signal was $<1 \%$ or $>99 \%$ of the total input range were excluded from the LFP analysis. This resulted in a slight decrease of the number of analyzed unaborted stimulus presentations per stimulus condition $(N)$ compared with the analyses of spiking activity (data for the main test: minimum/mean/maximum per stimulus $\times$ site combination across both monkeys and stimulus sets: $N($ standard $)=181 / 542 / 1032$, $N($ deviant $)=23 / 60 / 116, N($ reference $)=29 / 60 / 114$; median percentage of excluded unaborted stimulus presentations due to clipping of the LFP signal across conditions per stimulus $\times$ site combination $=0.69$ ).

To remove modulations of the LFP signal by the line noise $(50 \mathrm{~Hz})$ and the CRT monitor refresh rate $(60 \mathrm{~Hz})$, LFPs were sequentially filtered offline first with a digital $50 \mathrm{~Hz}$ notch filter $(48-52 \mathrm{~Hz}$ fourth-order Butterworth FIR filter; Fieldtrip Toolbox, F.C. Donders Centre for Cognitive Neuroimaging, Nijmegen, the Netherlands; http://fieldtrip. fcdonders.nl) and then with a $60 \mathrm{~Hz}$ notch filter $(58-62 \mathrm{~Hz}$ fourth-order Butterworth FIR filter). The spectral analysis of the LFPs was identical to that used in our previous studies (De Baene and Vogels, 2010; Kaliukhovich and Vogels, 2011, 2012) and was based on a time-frequency wavelet decomposition of the signal between 1 and $170 \mathrm{~Hz}$. By convolving the LFP signal for each stimulus presentation using complex Morlet wavelets (Tallon-Baudry and Bertrand, 1999) and taking the square of the convolution between the wavelet and signal, the time-varying power of the signal for every frequency was obtained. The complex Morlet wavelets had a constant center frequency-spectral bandwidth ratio $\left(f_{0} / \sigma_{f}\right)$ of 7 , with $f_{0}$ ranging from 1 to $170 \mathrm{~Hz}$ in steps of $1 \mathrm{~Hz}$. Averaging spectral maps (power as a function of frequency and time) across all analyzed presentations of a particular stimulus (A or B) per condition for each stimulus $X$ site combination produced the mean spectral maps per stimulus condition of that stimulus $\times$ site combination. The mean spectral maps of each stimulus $\times$ site combination were normalized at each frequency by the average power computed within a baseline window of $50 \mathrm{~ms}$ that ended at stimulus onset. For the normalization, the baseline power for the analyzed stimulus $\times$ neuron combination was computed by averaging across corresponding baselines for each of the three stimulus conditions of that stimulus $X$ neuron combination. Similar results were obtained when normalizing by the average baseline power per condition.

For further statistical analysis, we averaged for each stimulus $\times$ site combination the spectral power per stimulus condition within the following six time $\times$ frequency analysis windows: from 100 to $170 \mathrm{~Hz}$ within the time intervals (1) from 50 to $350 \mathrm{~ms}$ and (2) from 70 to $470 \mathrm{~ms}$ relative to stimulus onset, respectively; from 45 to $95 \mathrm{~Hz}$ within the time intervals (3) from 50 to $350 \mathrm{~ms}$ and (4) from 70 to $470 \mathrm{~ms}$; (5) from 18 to $38 \mathrm{~Hz}$ within the time interval from 35 to $135 \mathrm{~ms}$; and (6) from 4 to $15 \mathrm{~Hz}$ within the time interval from 15 to $185 \mathrm{~ms}$. These analysis windows were based on an inspection of the time-frequency power plots, averaged across the three stimulus conditions and each combination of subject and stimulus set, so as not to bias the selection of analysis windows. 
A

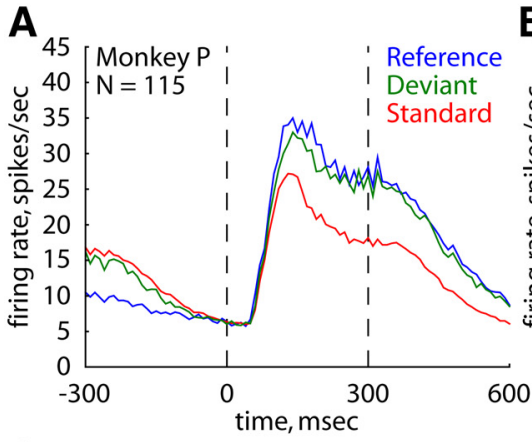

B
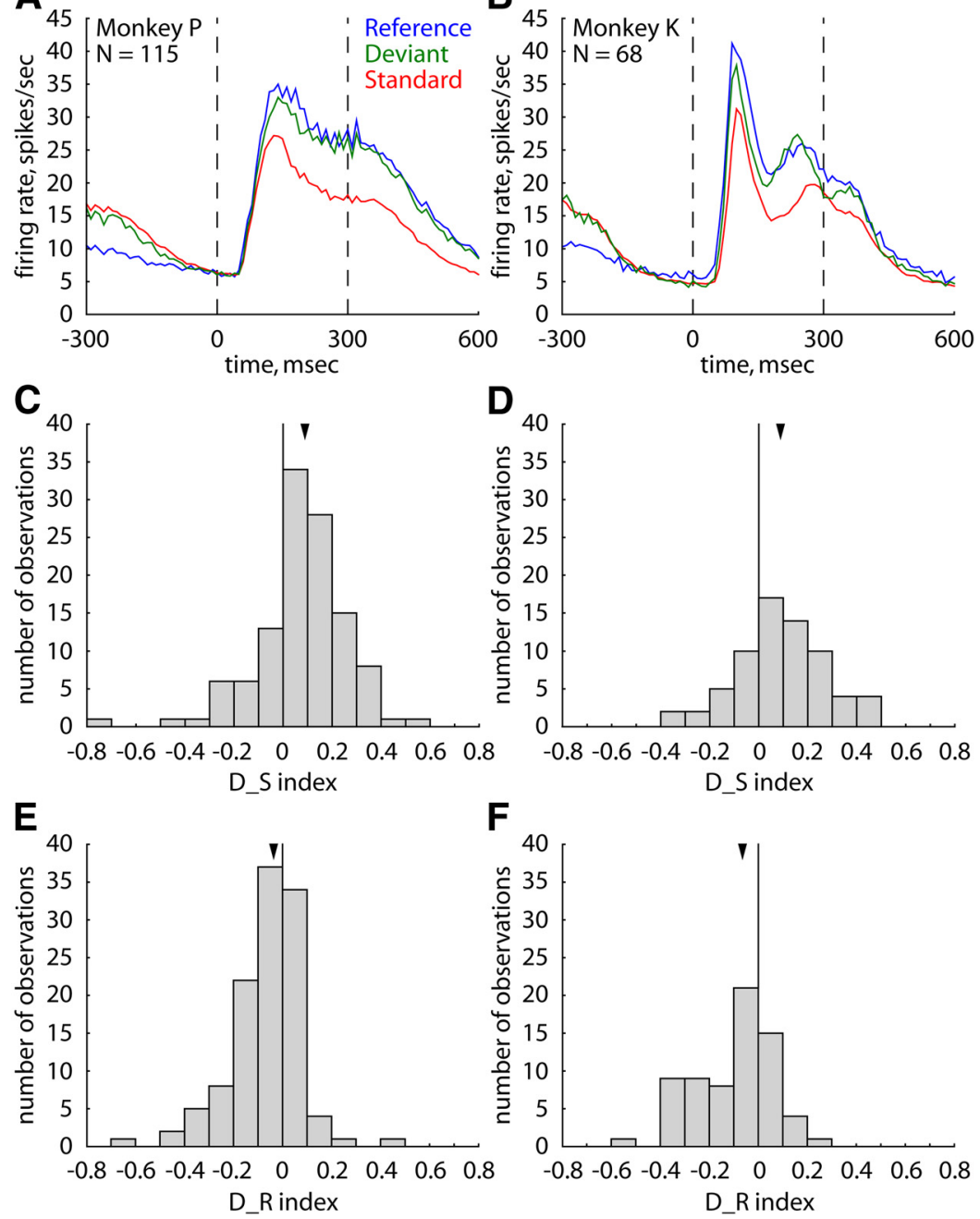

Figure 2. Effect of stimulus condition on the spiking activity for the natural stimuli. $A, B$, Population peristimulus time histograms for the natural stimuli for Monkeys $P(\boldsymbol{A})$ and $\mathrm{K}(\boldsymbol{B})$ in the main test (blocks of 100 stimulus presentations). Standard, Standard stimulus in oddball sequence; Deviant, the same stimulus presented as a deviant in the other oddball sequence; Reference, the same stimulus presented in the equiprobable condition. $N$ denotes the number of analyzed stimulus $\times$ neuron combinations. Stippled vertical lines indicate stimulus onset and offset. Bin width is $10 \mathrm{~ms}$. Mean activity is plotted at the center of the corresponding time bins (e.g., response at $0 \mathrm{~ms}$, stimulus onset, corresponds to the mean response in a time window from -5 to $5 \mathrm{~ms})$. (C, D) Distribution of the $D \_$S indices (comparing the responses to a stimulus presented as a deviant and as a standard; positive values indicate a higher response to a deviant compared with a standard stimulus) for the same stimulus $\times$ neuron combinations as in $\boldsymbol{A}, \boldsymbol{B}$ for Monkeys $\mathrm{P}(\boldsymbol{C})$ and $\mathrm{K}(\boldsymbol{D})$. $\boldsymbol{E}, \boldsymbol{F}$, Distribution of the $D \_R$ indices (comparing the responses to a stimulus presented as a deviant and as a reference; positive values indicate a higher response to a deviant compared with a referencestimulus) for the same stimulus $\times$ neuron combinations as in $\boldsymbol{A}, \boldsymbol{B}$ for Monkeys $P(\boldsymbol{E})$ and $K(\boldsymbol{F})$. Median values are indicated by an arrow. set to 0.017 (Bonferroni correction for a Type 1 error of 0.05). Most statistical analyses were performed separately for each of the two probe-stimuli (A and B; see Fig. 1), so that each neuron could contribute only a single data point per analysis. Only those neurons for which there was a significant response to the probe-stimulus were included in the analyses of that probe-stimulus.

\section{Results}

We recorded single-unit spiking activity and LFPs in anterior IT of two passively fixating monkeys to three sorts of stimulus sequences. In two oddball conditions, monkeys were presented sequences of two randomly interleaved stimuli, with each stimulus being presented either frequently (stimulus presentation probability $=0.9$; "standard") or rare (0.1; "deviant"). In the third equiprobable condition, the two stimuli ("reference") were randomly interleaved and presented with eight other stimuli, and all 10 stimuli were presented with an equal probability of 0.1 (Fig. 1). A vast amount of previous human ERP studies in several sensory systems demonstrated a stronger response to a deviant compared with the standard stimulus in an oddball sequence. This stronger response to the deviant could be attributed to repetition suppression or stimulus-specific adaptation. In addition, the higher response to the deviant stimulus in the oddball sequence may result from the discrepancy between the expected and the actually observed stimulus; i.e., reflect a surprise response (Barto et al., 2013). The latter "surprise" hypothesis predicts a stronger response to a stimulus presented as a deviant in the oddball sequence compared with the response to the same stimulus when presented in the equiprobable sequence (Schröger and Wolff, 1996). This is because in the equiprobable sequence stimulus expectations are less precise than in the oddball sequence in which a stimulus is presented with a high regularity (Lieder et al., 2013b).

Analysis of eye movements. For each analyzed stimulus $\times$ neuron combination, we computed the mean SD of the eye movement signal per stimulus condition along the $\mathrm{x}$ - and $y$-axes separately (Sawamura et al., 2006; De Baene and Vogels, 2010) in the same 50-350 ms analysis window and for the same stimulus presentations as those used for the analysis of spiking activity. The maximum difference between the mean SDs among the conditions was $<0.02^{\circ}$ and no consistent effects in the miniature eye movements were present across monkeys and stimulus sets, unlike the consistent effects observed for the neural responses. We also computed the mean (micro)saccadic frequency, peak velocity, and amplitude using the same method as Kaliukhovich and Vogels $(2011,2012)$, following Engbert and Kliegl (2003). Again, no consistent changes in saccadic frequency, peak velocity, and amplitude across conditions were present, arguing against eye movements causing the effect of stimulus condition on the neural response.

Statistics. When performing pairwise comparisons across the three conditions (standard, deviant, and reference), the significance level $p$ was
We first examine the responses of single IT units to a stimulus presented as a standard, deviant, or reference, followed by an analysis of the LFPs measured for the same conditions.

\section{Single-unit spiking activity}

Responses to stimuli in oddball and equiprobable sequences

Figure 2 shows the population peristimulus time histograms (PSTHs) for each stimulus condition and each monkey separately. Stimuli were complex "natural" images that are known to drive IT neurons well and that were selected for each neuron separately using a search test (see Materials and Methods). The number of responsive neurons recorded in Monkeys $\mathrm{P}$ and $\mathrm{K}$ was 66 and 34, respectively. For plotting the PSTHs, we selected only those stimulus $X$ neuron combinations in which the stimulus evoked a significant excitatory response in at least one of the three 
stimulus conditions (Monkey P: $N=115$; Monkey K: $N=68$; see Materials and Methods). In both animals, the mean response to the standard was lower than to the deviant and reference. The stimulus presented as a reference elicited the strongest mean response, with the response to a deviant stimulus being somewhat lower and in-between the responses to a standard and a reference. This is the opposite of a surprise response, because for the latter the response to the deviant should exceed the response to the reference. Note that the overlapping responses before stimulus onset for the standard and deviant conditions (Fig. 2) occurred because both these responses primarily reflected the later, sustained part of the response to a preceding standard stimulus. The smaller prestimulus response to the reference can be attributed to the choice of eight weakly effective stimuli in the equiprobable condition.

To perform statistical analyses of the differences in response to a stimulus for each of the three conditions, we computed the mean responses to a stimulus (averaged across presentations of that stimulus) per condition for each analyzed stimulus $\times$ neuron combination (see Materials and Methods). Then for the data of each monkey and probe-stimulus separately, we ran a Friedman ANOVA test with stimulus condition as repeated factor. In each monkey and for each probe-stimulus, we observed a highly significant effect of stimulus condition on the response (all $p$ values $<0.00001$ ), with the highest and lowest responses being elicited by a reference and a standard stimulus, respectively (median response across the two probe-stimuli, spikes/s: standard versus deviant versus reference: Monkey P: 16.8 vs 21.8 vs 23.0; Monkey K: 12.5 ves 15.9 vs 18.5). All the differences in response between conditions proved to be significant in each monkey when using Bonferroni correction for multiple comparisons (two-sided Wilcoxon matched pairs tests: all $p$ values $<0.01$ ), except for the difference between the reference and deviant stimuli for stimulus A in Monkey $\mathrm{P}$ (two-sided Wilcoxon matched pairs test, $p=0.023)$ and stimulus B in Monkey $\mathrm{K}(p=0.052)$.

To assess the variability of the effect of stimulus condition across stimulus $\times$ neuron combinations, we computed two contrast indices for each stimulus $\times$ neuron combination. The index $D \_S$ contrasted responses to the deviant and standard stimuli (Fig. 2C,D), with positive values indicating a stronger response to the deviant compared with the standard. The large majority (74\%) of the analyzed stimulus $\times$ neuron combinations showed a higher response to a deviant stimulus compared with a standard. The median $D \_S$ in each monkey was 0.09 , which indicates a $20 \%$ higher response to the deviant compared with the standard. The second index $D \_R$ compares the responses to the deviant and reference stimuli (Fig. $2 E, F$ ), with a positive value indicating a stronger response to the deviant compared with the reference, i.e., a surprise response. Only $33 \%$ of the stimulus $\times$ neuron combinations showed a positive $D \_R$ index. The median $D \_R$ was negative in each animal (Monkey P: -0.03 ; Monkey K: $-0.07)$, which is the opposite of what one would expect when there was a surprise response.

Further analyses showed that the above described effects of stimulus condition held irrespectively of how strongly a stimulus drove a neuron. Indeed, assigning stimulus $\times$ neuron combinations to 6 different groups based on the response strength to a stimulus presented as a reference (either using a range of 10 spikes/s per group or using an equal number of stimulus $\times$ neuron combinations per group) showed that in each group (except for the group with the lowest firing rates) the mean highest and lowest response was elicited by a reference and a standard stimulus, respectively (data not shown). Highly similar results were observed when equating the number of analyzed stimulus presentations across the three conditions by randomly drawing (without replacement) for each condition separately the minimum number of unaborted stimulus presentations across conditions from the pool of all unaborted stimulus presentations.

In the above experiment, the standard and deviant stimuli were chosen arbitrarily, with the only constraint being that at least one of the two stimuli drove a neuron. One could conjecture that to produce a surprise response in a neuron, the two stimuli need to engage interconnected neurons. Because many of our neurons responded to both stimuli (see below), this is likely to be the case for most of the sampled neurons. Nonetheless, we wished to address this issue more directly. Recently, Noudoost and Esteky (2013) reported that prolonged adaptation to a human face affects the responses of single macaque IT neurons to a face that differs by $>70^{\circ}$ in view from the adapted face, suggesting interconnections between neurons that respond to highly differing views. This neural view aftereffect was present even for IT neurons that were not face-selective. Given this finding of a neural aftereffect in IT for a face view that differs markedly from the adapter view, we assessed whether we could demonstrate a stronger response to a deviant compared with reference stimulus when the deviant is a face that differed in viewpoint by $90^{\circ}$ from the face used as the standard. Thus, we searched in Monkey P for IT neurons which were responsive to faces. We recorded 13 neurons that responded significantly to at least one view of a face (Fig. $3 A$; see Materials and Methods) while using the oddball and equiprobable sequences. In the oddball sequences, the stimuli were the frontal and profile views of the same face. As Noudoost and Esteky (2013), we did not require that the neurons were face-category selective or were located in an fMRI-defined face patch (Tsao and Livingstone, 2008). The results for the 24 significant face view $\times$ neuron combinations were qualitatively identical to those obtained for the natural stimuli (compare Figs. $2 A, B, 3 B$ ). Similarly, we observed a significant effect of stimulus condition on the response for each probe-stimulus separately (Friedman ANOVA tests: both $p$ values $<0.005$ ), with no evidence for a surprise response. Indeed, for the two probe-stimuli (A and $\mathrm{B}$ ) the mean response to a stimulus presented as a reference was higher (median across the two probe-stimuli: 17.3 spikes/s) than that when the same stimulus was presented as a deviant (median across the two probe-stimuli: 16.0 spikes/s; twosided Wilcoxon matched pairs test: stimulus A, $p=0.552, N=13$ neurons; stimulus B, $p=0.013, N=11$ neurons). The median $D \_S$ and $D \_R$ indices were 0.09 and -0.07 , respectively, which highly resembled those observed for the natural stimuli (see above). Due to this similarity, we pooled the data of the two types of stimuli in subsequent analyses.

A consistent feature of the data of both monkeys and the two stimulus sets is the higher response to the reference compared with the deviant stimulus. A potential explanation of the reduced response to the deviant is that many neurons responded to both the A and B stimuli that can produce cross-stimulus adaptation. Indeed, a response to both the standard and deviant stimuli may lead to a decreased response to the deviant because of crossstimulus adaptation (the adaptation effect due to the repeated presentation of the standard affecting the response to the deviant). Such a cross-stimulus adaptation-induced decreased response to the deviant may mask a potential surprise response to the deviant. To address this, we plotted the $D_{-} R$ indices as a function of the difference in net response between the two probestimuli A and B ( $B_{-} W$ index; see Materials and Methods). To do this, we took the $D \_R$ value only for one of the two probe-stimuli 
A

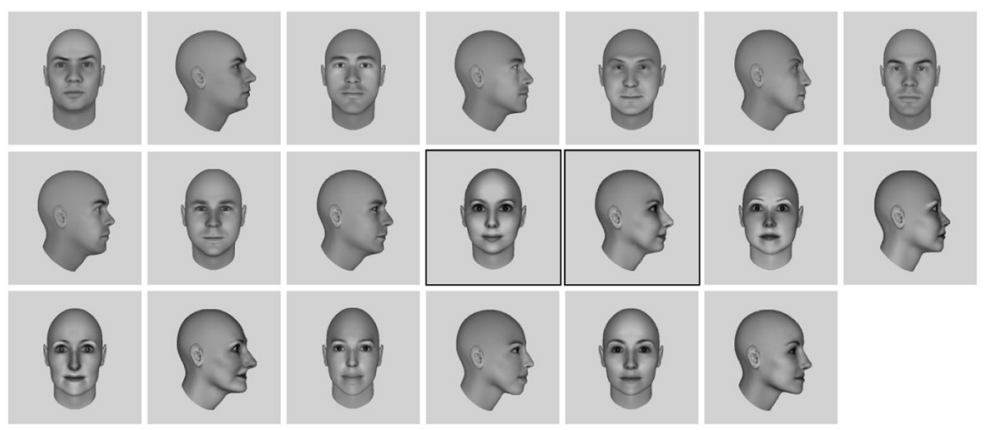

B

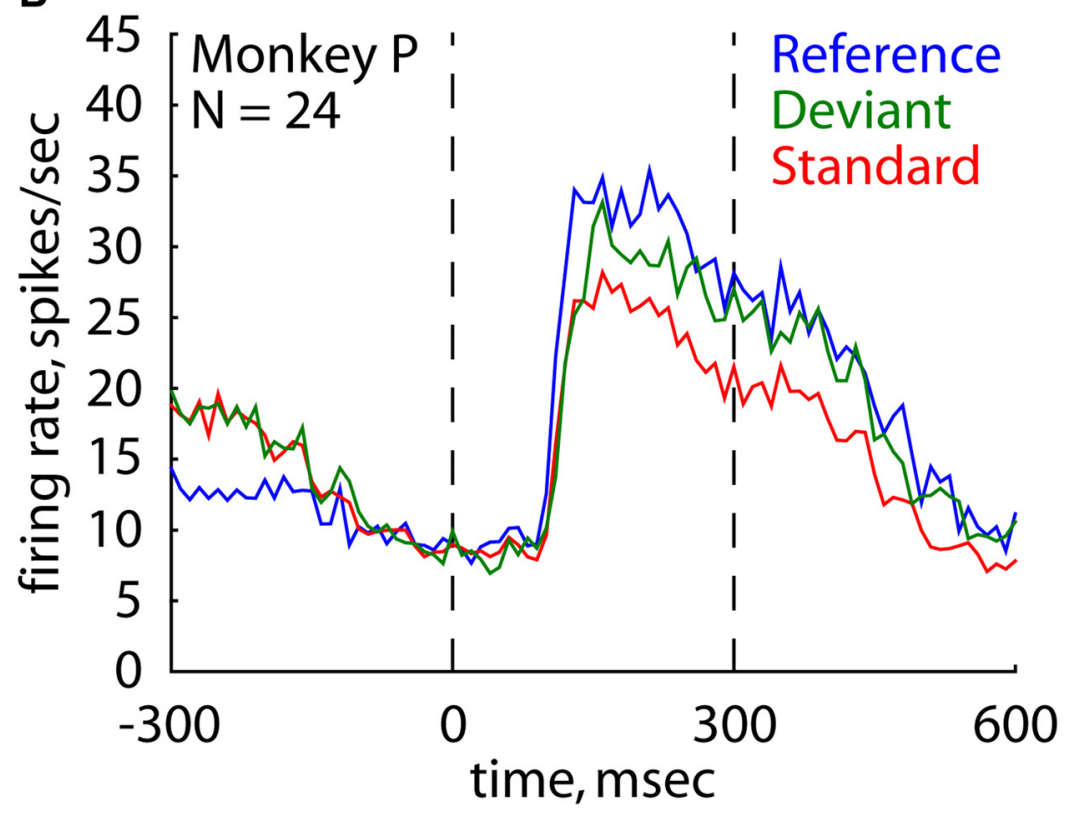

Figure 3. Effect of stimulus condition on the spiking activity for the face stimuli. $A$, Face stimulus set. The two boxes indicate the frontal and profile views of a female face. Such a pair of stimuli would be presented, when selected based on a search test, in the oddball and equiprobable sequences of 100 presentations. $B$, Population peristimulus time histogram for the face stimuli. $N$ denotes the number of analyzed stimulus $\times$ neuron combinations. Same conventions as in Figure $2 A$.

small tendency for a higher response to the deviant compared with the reference stimulus in the initial part of the response, but this tendency was negligible compared with the much larger and significant difference between the standard and deviant stimuli (Fig. 4C; two-sided Wilcoxon matched pairs test, $p<0.00001)$. Thus, no convincing evidence for a surprise response was present even in those cases in which cross-stimulus adaptation could not have masked a potentially enhanced response to the deviant.

For comparison, we plotted in Figure $4 B$ the responses to the "best" stimulus of a neuron in each of the three conditions for the group of neurons $(N=25$ neurons) that responded similarly to both probe-stimuli $\mathrm{A}$ and $\mathrm{B}\left(B \_W \leq 0.1\right)$. For those neurons, the response to the reference tended to be larger than that to the deviant. Note that despite the similar response to $\mathrm{A}$ and $\mathrm{B}$, the response to the standard was considerably smaller than that to the deviant (two-sided Wilcoxon matched pairs test, $p<0.0005$ ), which indicates substantial stimulus-specific adaptation. This agrees with the Sawamura et al. (2006) results, showing the same effect as theirs but for an oddball sequence.

\section{Response as a function of presentation order in a sequence}

In the previous analyses, the responses to a stimulus were computed by averaging the responses to that stimulus regardless of the presentation order of the stimulus within a block of stimuli. However, this conventional technique to calculate the response to a stimulus in the oddball sequences ignores the temporal evolution of the response within a block. To compare

(A or B) to which the neuron responded best when presented as a reference. Thus, each neuron contributed a single data point. As shown in Figure 4, our neurons showed a wide variation in the $B \_W$ index, with some neurons responding similarly to both probe-stimuli $\mathrm{A}$ and $\mathrm{B}$ ( $B_{-} W$ close to 0$)$ and the others responding to only one probe-stimulus ( $\mathrm{A}$ or $\mathrm{B} ; B_{-} W=1$ ). Note that there was a weak trend toward an increase of the $D_{-} R$ index with increasing $B \_W$, as expected if there was cross-stimulus adaptation of the response to the deviant due to repeated presentations of the standard stimulus (Fig. 4A, regression line in red). However, the Pearson correlation coefficient between $D \_R$ and $B \_W$ failed to reach significance $(r=0.14, p=0.15, N=113)$.

Cross-stimulus adaptation of the deviant cannot occur when there is no response to the adapter, i.e., the standard stimulus (Sawamura et al., 2006). Thus, for neurons for which $B \_W$ is equal or close to 1 , no masking of a potential surprise response by cross-stimulus adaptation could be present. However, neurons with $B \_W \geq 0.9$ did not show a significantly higher response to the deviant compared with the reference $(N=27$ neurons; median response: deviant: 21.8 spikes/s; reference: 21.5 spikes/s; two-sided Wilcoxon matched pairs test, $p=0.24$ ). There was a the temporal course of the responses within a block among the three stimulus conditions, we computed the population average time course of the response to a stimulus within a block (sequence of 100 presentations) for each stimulus condition separately (Fig. 5A; see Materials and Methods). Because the results observed in both monkeys were similar, we pooled the analyzed stimulus $\times$ neuron combinations across both monkeys and stimulus sets. Note that the number of data points for each presentation order varies across stimulus conditions. In all three conditions, the highest response was observed for the first presentation within a block. Then there was a sharp decline in response with presentation order for the standard stimulus, whereas such a decline in response was limited to the first few trials for the deviant and reference stimuli. Importantly, the average response to the deviant stimulus was stable after the first few trials and there was no trend toward a stronger response to the deviant compared with the reference stimulus with increasing presentation order.

To quantitatively compare the time courses among the three stimulus conditions, we fitted each time course with a polynomial inverse first order function and performed bootstrapping to de- 
A

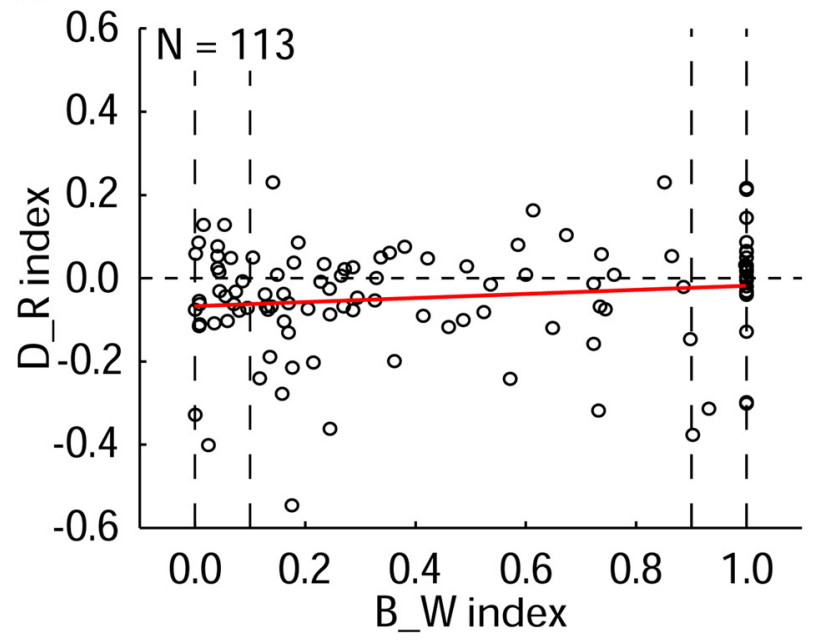

B
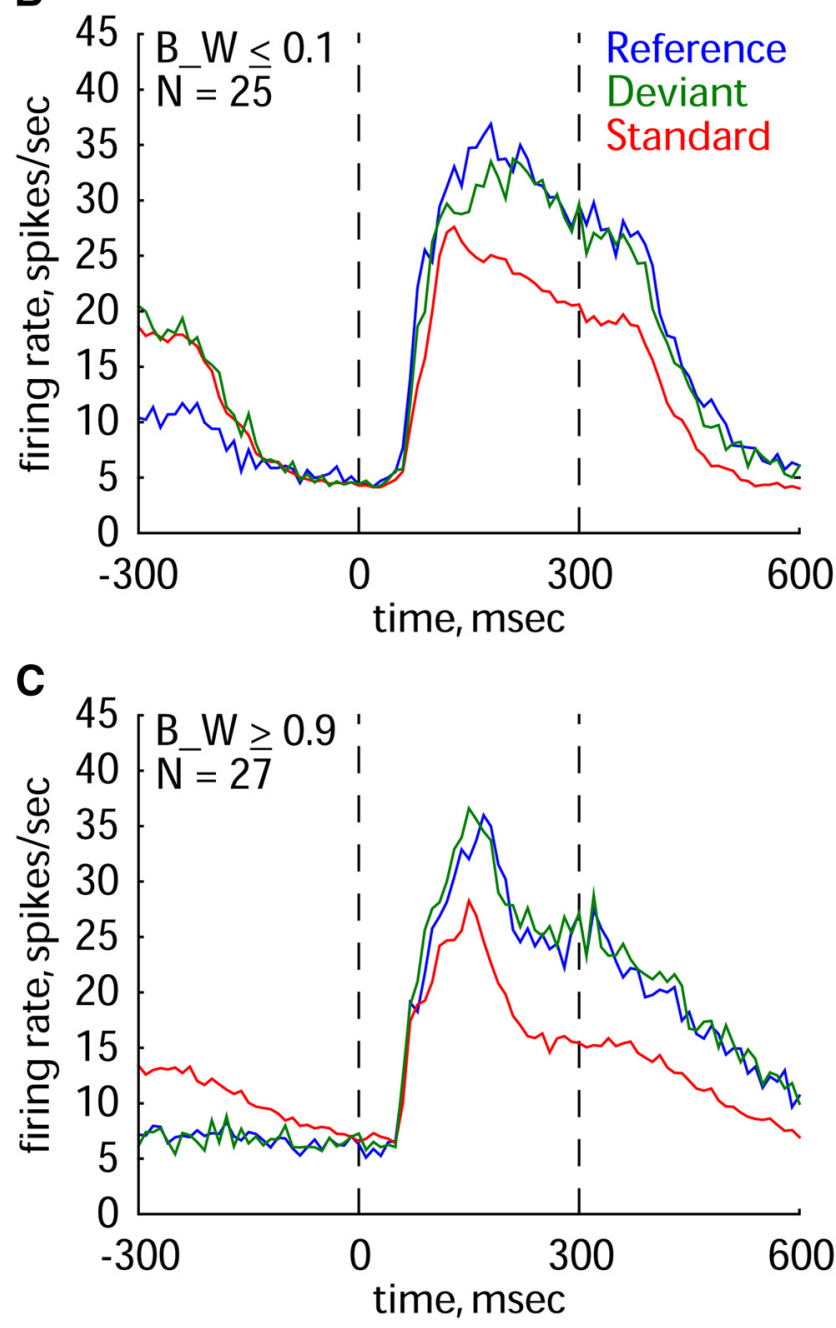

Figure 4. Effect of the difference in response to the two probe-stimuli $A$ and $B$ ( $B \_$W index) on the response in oddball and equiprobable sequences. $A, D \_R$ index plotted as a function of the $B \_W$ index for each of the 113 neurons tested in the main test (100 stimulus presentations per block) for both monkeys and stimulus sets. Each point corresponds to the $D$ R value for one of the two stimuli ( $A$ or $B$ ) to which a neuron responded best when presented as a reference. The linear regression line is indicated in red. $\boldsymbol{B}$, Population peristimulus time histograms for the 25 neurons with $a$ $B \_W$ index $\leq 0.1$ ( $\boldsymbol{A}$, left pair vertical stippled lines). $\boldsymbol{C}$, Population peristimulus time histograms for the 27 neurons with a $B \_W$ index larger or equal to $0.9(\boldsymbol{A}$, right vertical stippled lines). Same conventions as in Figure $2 A$. termine the significance of the difference between the fitted parameter values of the three conditions (see Materials and Methods). The parameter describing the asymptote (parameter $a$; see Materials and Methods) was significantly higher for the reference $(a=26.2)$ compared with the deviant stimulus ( $a=$ 23.9; bootstrap test; see Materials and Methods). The asymptote for the standard stimulus was the lowest $(a=18.3)$ and differed significantly from those for the deviant and reference stimuli, thus, paralleling the results observed when the responses were averaged across presentation order (Fig. 2). The parameter describing the decay size (parameter $b$ ) was the largest for the standard stimulus $(b=16.2)$ and significantly differed from those for the deviant $(b=5.4$; bootstrap test $)$ and reference $(b=3.4)$ conditions. The difference in decay size between the latter two conditions failed to reach statistical significance. These data agree with strong stimulus-specific repetition suppression for the standard stimulus, whereas the responses to the deviant and reference stimuli were stable throughout a block of stimuli, except for the first few presentations.

Figure $5 B$ shows the population PSTHs for the three stimulus conditions of the 27 neurons for which $B \_W \geq 0.9$ and this for the last $25 \%$ of the presentations of a block of stimuli (presentation order 76-100). Again, no evidence for a surprise effect was present. Thus, we did not miss a surprise effect by pooling across all stimulus presentations of a block, which is the usual practice in ERP studies of the mismatch negativity.

Control experiment: sequences of 300 stimulus presentations Although the responses to the deviant and reference were relatively stable after the first few presentations in a sequence (Fig. $5 A$ ), it was still possible, although unlikely, that sequences consisting of 90 presentations of the standard stimulus were insufficient to build up an expectation of a stimulus during passive fixation. To address this question, we additionally recorded the responses of $30 \mathrm{IT}$ neurons (Monkey $\mathrm{P}$, natural stimuli) using sequences of 300 presentations with the same stimulus presentation probabilities as in the 100-presentation-long sequences (main test). The order of the conditions was carefully counterbalanced across neurons to control for potential condition order effects. Similar to the main test, we pooled the responses to the stimuli of a single sequence across the responsive stimulus $X$ neuron combinations $(N=43)$. We found no significant difference in response between the deviant (median across the two probe-stimuli: $21.2 \mathrm{spikes} / \mathrm{s}$ ) and the reference stimuli for each probe-stimulus (Fig. $6 \mathrm{~A}$; median across the two probe-stimuli: 23.7 spikes/s; two-sided Wilcoxon matched-pairs tests, both $p$ values $>0.35$ ). The response to the standard (median across the two probe-stimuli: 17.2 spikes/s) was significantly lower than for the other two stimulus conditions for one of the two probestimuli (stimulus A: both $p$ values $<0.0001, N=25$ neurons; stimulus B: both $p$ values $>0.24, N=18$ neurons). Note that the lack of significant differences in response across the conditions for stimulus B could be explained by the selection bias of the two probe-stimuli during the recordings of the 300-presentationlong sequences. Specifically, stimulus A was usually selected to be the most effective stimulus (median response when presented as a reference: 30.1 spikes/s). On the contrary, stimulus B was selected to evoke a weaker or no response (median response when presented as a reference: 12.5 spikes/s). Importantly, highly similar effects were observed when comparing the data of the first 100 (Fig. 6B) and the last 100 stimulus presentations (Fig. 6C) of the 300-presentation-long sequences. This indicates that adding more presentations of the standard stimulus to the 90 presenta- 
A
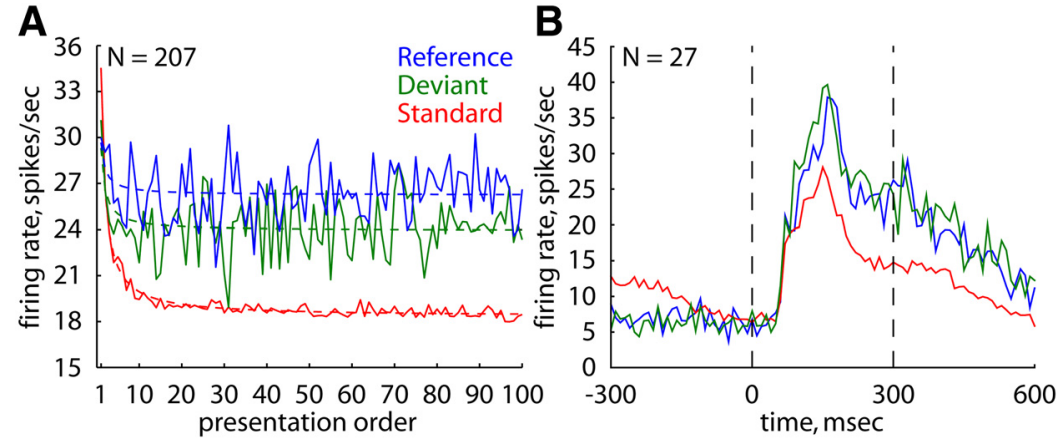

Figure 5. Effect of presentation order within a block of stimuli on the response for the three stimulus conditions. $A$, The time course of the average population response to a stimulus within a block (sequence of 100 stimulus presentations) plotted for each condition separately. Data of the analyzed stimulus $\times$ neuron combinations $(N=207)$ are pooled across both monkeys and stimulus sets. Note that the number of data points for each presentation order varies across stimulus conditions. The stippled curves correspond to fits of the time courses with a polynomial inverse first order function. $\boldsymbol{B}$, Population peristimulus time histograms of the mean responses for the three conditions of the 27 neurons for which $B \_W \geq 0.9$ and this for the last $25 \%$ of the stimulus presentations of a block (presentation order $76-100)$. Same conventions as in Figure $2 A$.

tions of that stimulus already present in the main test did not change the observed results, that is, an absence of a surprise effect to the deviant. To control for possible cross-stimulus adaptationinduced reductions of the response to the deviant, we analyzed the neurons $(N=21)$ for which $B_{-} W \geq 0.90$ and that were tested with the 300-presentation-long sequences. As before, we found no evidence in support of a surprise response to the deviant for these neurons (median response to the deviant and the reference stimuli: 31.8 and 32.4 spikes/s; two-sided Wilcoxon matched-pairs test, $p=0.48$ ).

To conclude, single macaque IT neurons showed the expected stimulus-specific repetition suppression for the repeated standard stimuli, whereas there was no evidence for an enhanced or "surprise" response to the deviant in the oddball sequences.

\section{Effects of stimulus history on IT responses}

Previous studies of adaptation in macaque IT used either short stimulus sequences (Gross et al., 1967, 1969; Baylis and Rolls, 1987; Miller et al., 1991b; Liu et al., 2009) or long sequences but with repetition of a single stimulus (Miller et al., 1991a; Sawamura et al., 2006). Unlike those previous designs, oddball sequences give us the opportunity to investigate the effect of the local stimulus history on stimulus-specific adaptation. Such analyses of responses in oddball sequences have been conducted before in the auditory cortex of anesthetized cats (Ulanovsky et al., 2004) and the inferior colliculus of anesthetized rats (Zhao et al., 2011). These studies distinguished local effects, i.e., of the just preceding stimuli within a sequence, and global effects, i.e., of the overall presentation probability, on the responses. The effect of local stimulus history can be examined by averaging the responses to a stimulus as a function of the preceding stimulus sequence and that with increasing order of the examined stimulus sequence. For instance, one can compare the responses to stimulus A when this stimulus followed AA versus when it followed BA; i.e., AAA versus BAA. We performed such analysis (see Materials and Methods) on the responses of the main test (blocks of 100 stimulus presentations), normalized by the mean response to that stimulus in the equiprobable condition. The normalization allowed us to combine the data of both monkeys and stimulus sets. The tree diagrams in Figure 7 show the mean normalized response (and bootstrapped 95\% confidence intervals) to a stimulus when it is a standard (high probability presentations; Fig. $7 A$ ) and deviant (low probability presentations; Fig. $7 B$ ) as a function of the local history in that sequence. Only averages of at least 100 unaborted stimulus sequences are shown.

The average response to a stimulus depended strongly on the just preceding stimulus (BA vs AA), and this effect was even present for high probability stimuli (Fig. 7A, second order), indicating fast stimulus-specific adaptation effects. A stimulus three presentations back in time affected the response because the response to ABBA was lower than to BBBA (Fig. $7 B$, fourth order), in line with previous observations that stimulus-specific adaptation can bridge an intervening stimulus (Miller et al., 1991b; Sawamura et al., 2006). Finally, the response to the same fourth order sequence (e.g., BABA) tended to differ between the low and high probability sequences, suggesting an effect of the overall stimulus probability or a mechanism with at least a "memory" span of four items. Overall, this analysis suggests that IT responses in an oddball sequence are strongly affected by stimulusspecific adaptation effects that operate with a relatively short stimulus history, with weaker influences of effects that operate at longer time scales.

\section{Effect of fixation breaks on responses to the standard}

The monkeys broke fixation during the sequences, which interrupted the stimulus presentation of the sequence. The mean number of consecutive stimulus presentations in unaborted sequences of the main test was 8.5 (averaged across all sequences, monkeys, and stimulus sets). The median fixation break duration, defined as the time interval between the stimulus offset before the fixation break and the stimulus onset after the fixation break, was $1404 \mathrm{~ms}$. An evident question is how these fixation breaks affected the responses within a sequence. This is of a particular interest because the above stimulus history analysis data are in line with adaptation processes that act relatively fast. To assess the effect of fixation breaks on the responses, we compared the responses to a stimulus before and after a fixation break. This was only possible with sufficient statistical power for the standard stimuli because few deviants happened to be presented just before and after a fixation break. Figure $8 \mathrm{~A}$ plots the mean response to the standard before and after a fixation break as a function of the fixation break duration (running average with width of a boxcar kernel $500 \mathrm{~ms}$ with a step of $10 \mathrm{~ms}$ ) for all standards that were followed by a fixation break, pooled across all analyzed stimulus $\times$ neuron combinations of both monkeys and stimulus sets. The mean response to the standard was significantly higher after than before the fixation break (two-sided Wilcoxon matchedpairs test, $p=5.1594 \times 10^{-217}$; pooled across the 7259 fixation breaks) and the response to the standard after the fixation break increased with fixation break duration (Spearman rank correlation: $r=0.98, p<0.0005$; computed using nonoverlapping bins of $500 \mathrm{~ms})$. Thus, a fixation break weakened the effect of the preceding stimuli on the response to the standard stimulus that follows that break. However, it did not fully reset the latter effect since the mean responses after the fixation break were still below those to the first presentation of the standard in the same block of stimuli (Fig. 8 A, blue curve).

One could argue that the presence of fixation breaks interrupts the expectation of a stimulus. Indeed, if a potential surprise 

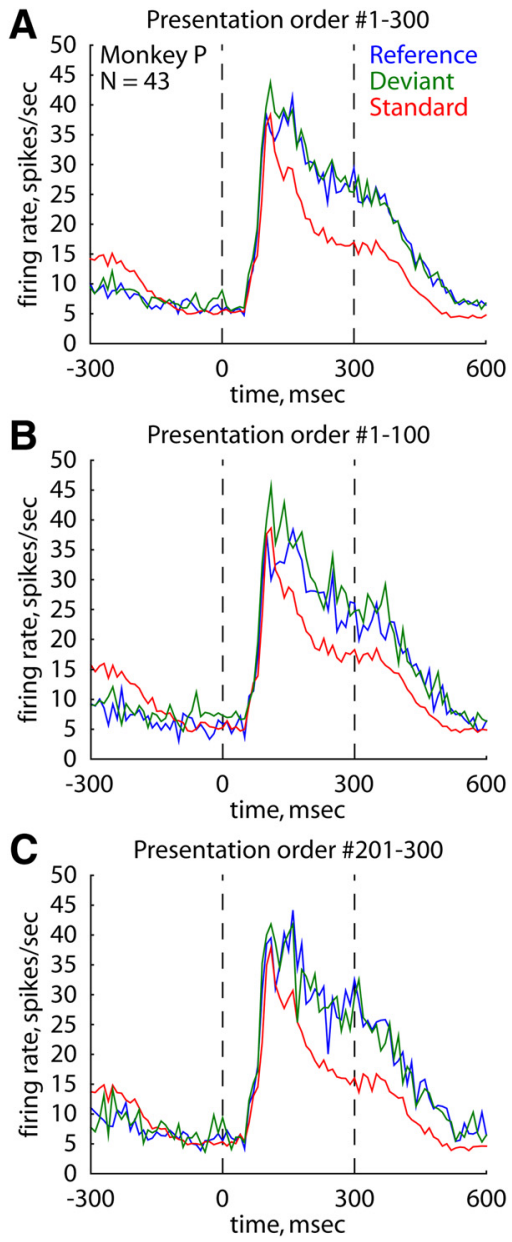

Figure 6. Spiking activity within blocks of 300 stimulus presentations. A, Population peristimulus time histograms of the responses for the three stimulus conditions, averaged across the presentations of the whole block of stimuli. $B, C$, Population peristimulus time histograms of the responses for the three stimulus conditions, averaged across the first $100(\boldsymbol{B})$ or the last 100 presentations $(\boldsymbol{C})$ of the 300 -presentation-long blocks. Data obtained in Monkey P (43 stimulus $\times$ neuron combinations). Same conventions as in Figure $2 A$.

response develops only during an unaborted sequence, the development of such a response will need to start anew after each break. Although very unlikely, the absence of the surprise response in the previous analyses might have arisen due to these interruptions in the expectation. To address this question, we computed the mean responses to a stimulus presentation for each unaborted sequence as a function of the presentation order within that unaborted sequence. For the presentations of a standard, we averaged the responses to all standards as a function of their presentation order. For a deviant stimulus, we averaged the responses to deviants as a function of the number of preceding standards in that unaborted sequence. Thus, a mean response to a deviant or a standard at presentation order 3 corresponds to the response to a deviant or a standard, respectively, following two presentations of the standard stimulus. The responses to the same stimuli presented as a reference were averaged as a function of their presentation order within unaborted sequences of the equiprobable condition. Averaging was performed first per stimulus $X$ neuron combination and then across the stimulus $\times$ neuron combinations (averaging across stimulus presentations or averaging of normalized responses produced highly similar results). Figure $8 B$ shows the average response to a standard, deviant, and reference as a function of the presentation order within
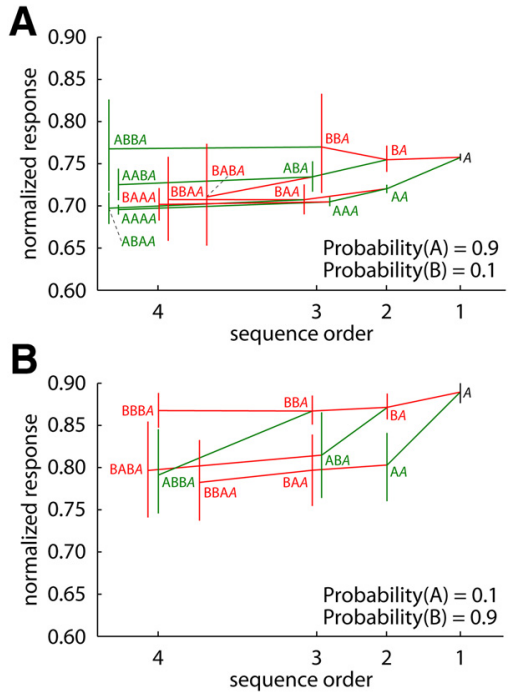

Figure 7. Effect of stimulus history on spiking responses to a stimulus in oddball sequences. Tree diagrams show the mean normalized response (and bootstrapped 95\% confidence intervals) to a stimulus, presented as a standard (high probability presentations; $\boldsymbol{A}$ ) or a deviant (low probability presentations; $\boldsymbol{B}$ ), as a function of the local history in that sequence. The responses are plotted as a function of sequence order, i.e., the number of stimuli taken into account to define the sequence. The local stimulus history is indicated for each averaged response (e.g., BA corresponds to the response to stimulus $A$ following $B, A B B A$ corresponds to the response to stimulus $A$ following the sequence $A B B$ etc.). Green symbols indicate responses to $A$ in sequences where the first stimulus of that short sequence was the same, i.e., $A$, whereas red symbols indicate responses to $\mathrm{A}$ in sequences where the first stimulus was different, i.e., B. Green lines connect sequences of which the higher-order sequence starts with A, i.e., is identical to the stimulus for which the response is averaged, whereas red lines connect sequences of which the higher-order sequence starts with B. Only averages of at least 100 unaborted stimulus sequences are shown. Data were obtained in the main test.

an unaborted sequence, with presentation order 1 corresponding to the presentation of the stimulus immediately after a fixation break. The data of all stimulus $\times$ neuron combinations of both monkeys and stimulus sets were pooled. To have sufficient power, only averages of at least 50 stimulus $\times$ neuron combinations are plotted, explaining why we limited the analysis to a within-sequence presentation order of maximum 15 . The first observation is that the mean response to the deviant is greater than that to the standard for stimuli presented immediately after a fixation break (two-sided Wilcoxon matched-pairs test, $p<$ 0.0000001; $N=207$ stimulus $\times$ neuron combinations). This indicates that the sequence before the fixation break influences the responses after the fixation break, i.e., there is a memory of the previous stimulus sequence. The second observation is that there is a decrease of the mean response with increasing presentation order after the fixation break. This was the case for the standard (Spearman rank correlation of presentation order and mean response: $r=-0.85, p<0.00005 ; N=15)$ and less so for the deviant stimulus $(r=-0.63, p<0.05)$. Note that the response to the deviant tended to decrease with increasing presentation order, unlike what one would expect from a surprise response. Indeed, repetition of the standard is supposed to increase the precision of the prediction (Lieder et al., 2013a) and surprise defined as the degree to which the expectation needs to be updated by the current stimulus will increase with the number of standards (Ostwald et al., 2012), both leading to an increased response to the deviant as a function of the number of standards. Furthermore, the difference in response between the deviant and reference condition appeared stable throughout the unaborted sequence. 
A

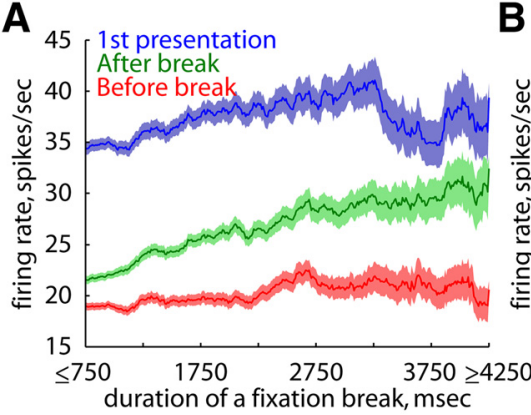

B

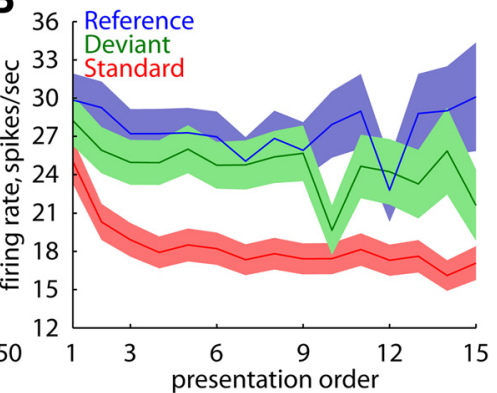

Figure 8. Effect of fixation breaks on spiking activity for the three stimulus conditions (main test; blocks of 100 stimulus presentations). $\boldsymbol{A}$, The mean response to the standard before (red) and after a fixation break (green) as a function of the fixation break duration (running average with width of a boxcar kernel of $500 \mathrm{~ms}$ with a step of $10 \mathrm{~ms}$ ) for all standards that were followed by a fixation break, pooled across all analyzed stimulus $\times$ neuron combinations of both monkeys and stimulus sets. The blue curve indicates the mean responses to the first presentation of the standard for the same blocks of stimuli as those of the other curves. Note that the sample sizes were smaller for the longer fixation break durations. $\boldsymbol{B}$, The mean responses to a stimulus presentation for each unaborted sequence plotted as a function of the presentation order within that unaborted sequence, with presentation order 1 corresponding to the presentation of the stimulus immediately after a fixation break. For the standard condition (red), the responses to all standards were averaged as a function of their presentation order. For the deviant condition (green), the responses to deviants were averaged as a function of the number of preceding standards in that unaborted sequence. Thus, a mean response to a deviant or a standard at presentation order 3 corresponds to the response to a deviant or standard, respectively, following two presentations of the standard stimulus after a fixation break. The responses to the same stimuli presented as a reference (blue) were averaged as a function of their presentation order within unaborted sequences of the equiprobable condition. Averaging was first performed for each analyzed stimulus $\times$ neuron combination and then across all analyzed stimulus $\times$ neuron combinations. The data of both monkeys and stimulus sets were pooled. Only averages of at least 50 stimulus $\times$ neuron combinations are plotted. Bands indicate SEM.

\section{LFPs}

In addition to single-unit spiking activity, we simultaneously recorded LFPs which presumably reflect neuronal activity within the local cortical network (Mitzdorf, 1987; Logothetis, 2003; Buzsáki et al., 2012; Einevoll et al., 2013). To specify the time $\times$ frequency windows used for the analysis of LFPs spectral power, we first averaged the normalized mean spectral maps across conditions for each analyzed stimulus $\times$ site combination and then averaged the thus computed spectral maps per stimulus $\times$ site combination across the same selections of stimulus $\times$ neuron combinations as those used for the analyses of spiking activity of the main test (100-presentation-long sequences). Using the stimulus-induced changes in power, averaged across the three stimulus conditions, we defined six time $\times$ frequency analysis windows (see Materials and Methods) for further analysis. An additional constraint when defining these analysis windows was the presence of consistent changes in power across both monkeys and stimulus sets. Note that we specified two time intervals for the gamma frequency range. The time interval of 50-350 ms was identical to that used in the analyses of spiking activity, whereas the second interval of 70-470 ms matched better the temporal span of the increase in power with stimulus presentation for this frequency range.

To quantify the effect of stimulus condition on the LFPs spectral power, we averaged the normalized mean spectral power across the analyzed stimulus $\times$ site combinations for each stimulus condition and each of the six time $\times$ frequency analysis windows separately (Fig. 9). For further statistical analysis, we ran for each of the two probe-stimuli separately a Friedman ANOVA test with stimulus condition as repeated factor. These ANOVAs were performed for each time $\times$ frequency window and combination of monkey and stimulus set. For the natural stimuli, both monkeys showed a significant effect of stimulus condition for the gamma frequency power (time $\times$ frequency windows nos. 1, 2, 3, and 4; see Materials and Methods; for the two probe-stimuli A and $\mathrm{B}$ : all $p$ values $<0.0001$ ) with the lowest response being to the standard stimulus. The responses to the reference and deviant stimuli for these high frequencies were significantly higher than that to the standard stimulus for each of these four time $X$ frequency analysis windows after Bonferroni correction (two-sided Wilcoxon matched pairs tests: all $p$ values $<0.017$ ), with no difference between the reference and the deviant stimuli. For the face stimuli in Monkey $\mathrm{P}$, there was a significant modulation of the spectral power by stimulus condition for both time intervals of the two gamma frequency bands, but this was only for one of the two probestimuli (time $\times$ frequency windows nos. 3 and 4; Friedman ANOVA tests: all $p$ values $<0.05$ ). Similar to the natural stimuli, that probe-stimulus presented as a reference tended to evoke the highest response, which differed from the response to the same stimulus when presented as a standard. The response to the deviant stimulus was in-between the responses to the reference and standard stimuli (Fig. 9). Thus overall, the effect of stimulus condition on gamma power was very similar to that observed for the spiking data (compare Figs. 2, 9).

For the $18-38 \mathrm{~Hz}$ frequency band (Fig. 9; time $\times$ frequency window no. 5), Monkey $\mathrm{P}$ showed similar trends in response across the three stimulus conditions for both stimulus sets, with this modulation being significant for the natural stimuli (Friedman ANOVA tests: for each probe-stimulus, $p<0.05$ ) and for one of the two face probe-stimuli $(p<0.02)$. In this monkey, the response to the standard was similar to that to the reference and these responses were both higher than the response to the same stimulus when presented as a deviant (Fig. 9). This could be interpreted as a surprise-related response although with an opposite sign (weaker instead of stronger response to the deviant stimulus). However, such an effect was not present in Monkey K. Although in this animal the power in this lower-frequency band was also significantly modulated by stimulus condition (Friedman ANOVA test: for each probe-stimulus, $p<0.005$ ), the only significant differences were between the reference and the other two conditions (all $p$ values $<0.005$ ).

For the $4-15 \mathrm{~Hz}$ frequency band (Fig. 9; time $\times$ frequency window \#no. 6) in Monkey $\mathrm{P}$, there was a significant effect of stimulus condition for only one of the two face probe-stimuli (Friedman ANOVA test, $p<0.05$ ) and not for the natural stimuli. Contrary to Monkey P, Monkey K showed a significant modulation of the response by the stimulus condition (Friedman ANOVA test: for each probe-stimulus, $p<0.00001$ ) for the natural stimuli, with all the differences in response between the three conditions being pairwise significant after Bonferroni correction (all $p$ values $<0.0005$ ). Thus, for the frequencies $<38 \mathrm{~Hz}$ we found no consistent effects of stimulus condition on the LFPs spectral power. The latter contrasts with the consistent results obtained for the spiking data (compare Figs. 2, 3) and gamma LFPs power.

These effects of stimulus condition on the LFPs spectral power could not be attributed to the different number of analyzed stimulus presentations per condition. This was confirmed by running 
the same analyses after equating the number of analyzed stimulus presentations across conditions for each stimulus $X$ site combination and observing qualitatively identical effects of stimulus condition.

\section{Discussion}

We observed a consistent stimulusspecific adaptation effect in visual oddball sequences in macaque IT cortex with reduced responses to the standard compared with both the deviant and the same stimulus when presented in an equiprobable sequence. This adaptation effect was present at the single-unit level as well as for LFP power $>50 \mathrm{~Hz}$. However, we did not find any evidence for a surprise response to a deviant stimulus, neither for single units nor for LFP signals of different frequency bands.

What could be the cause(s) of the absence of a surprise response in monkey IT, given the presence of such responses in human ERPs, the visual mismatch negativity (vMMN; for review, see Kimura, 2012)? It is unlikely that the choice of complex stimuli in our study caused the absence of a surprise response. Although originally reported for simple visual features, recent studies have demonstrated the vMMN for complex stimuli, such as symmetric patterns (Kecskés-Kovács et al., 2013a), facial expression (Astikainen and Hietanen, 2009; Kimura et al., 2012; Li et al., 2012; Stefanics et al., 2012) and face gender (Kecskés-Kovács et al., 2013b).

A second factor that needs to be taken into consideration is the presence of interruptions in the sequences due to fixation breaks. We do not believe that the fixation breaks can explain the absence of a surprise effect. First, the suppressed responses to the standard survived the fixation breaks (Fig. 8A). Furthermore, the difference between standard and deviant was present in the very first presentation following a fixation break (Fig. 8B). Both observations indicate a memory of the stimulus sequence from before the fixation break. Second, the vMMN in humans survives breaks of the stimulus sequence of 5-12 s (Maekawa et al., 2009; Kimura, 2012), intervals much longer than our median fixation break duration. Also, a face gender vMMN was shown using a mean interstimulus interval of 2250 ms (Kecskés-Kovács et al., 2013b). Third, the vMMN can be elicited using short sequences of five stimuli (Kimura et al., 2006). In fact, "roving-standard" paradigms in which standards are presented in short trains and change between successive trains (e.g., aaaabbbbbbcccc...) are becoming popular to examine prediction-related responses (auditory modality: Cowan et al., 1993; Winkler et al., 1996; Garrido et al., 2008; Costa-Faidella et al., 2011; Lieder et al., 2013a; Szycik et al., 2013; vMMN: Sulykos et al., 2013). Furthermore, according to a recent model based on predictive coding such short sequences are expected to generate a

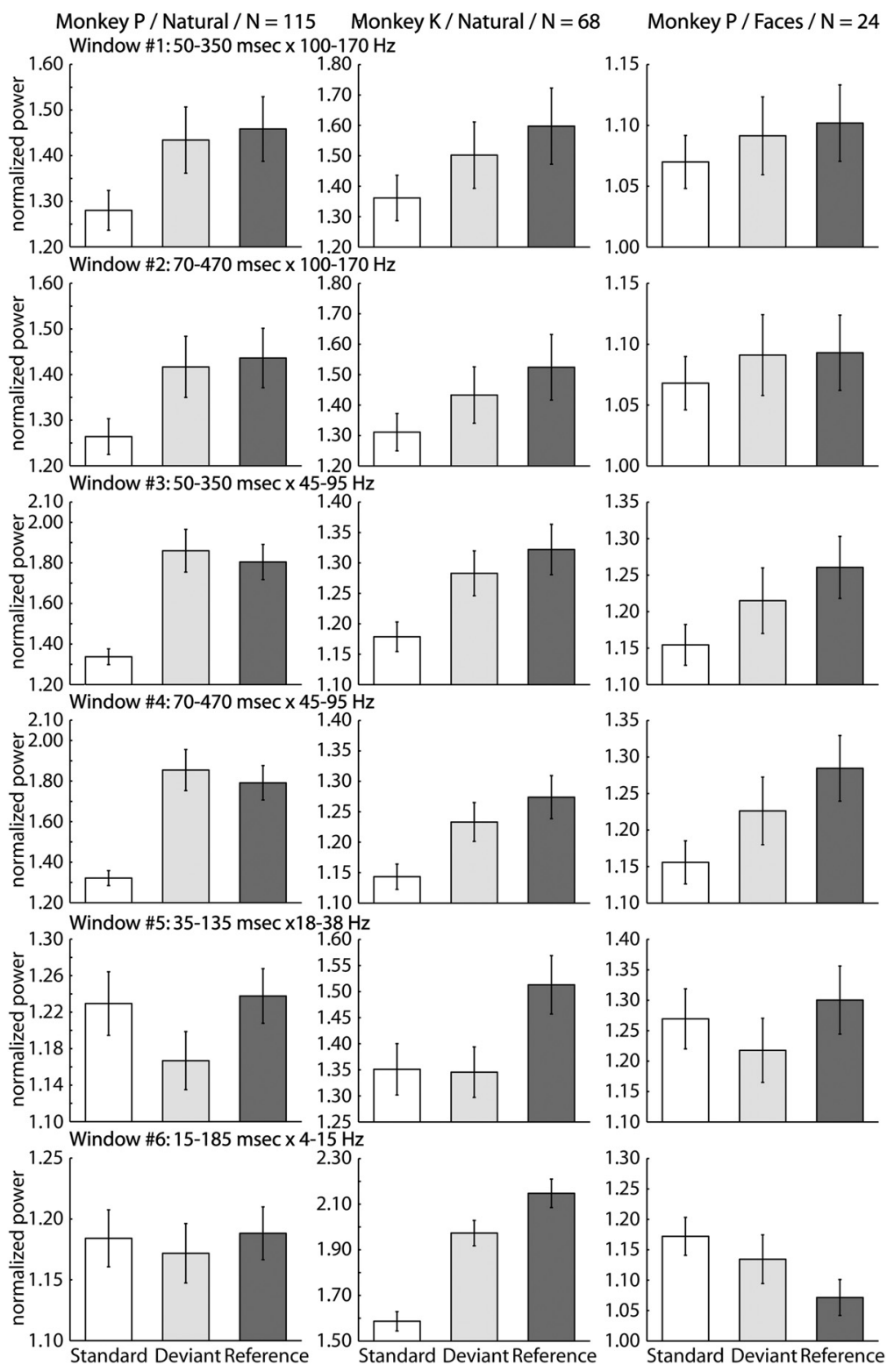

Figure 9. LFP power for the three stimulus conditions in oddball and equiprobable sequences (main test; 100-presentationong sequences). Mean normalized power (and SEM; averaged across the two probe-stimuli) for each stimulus condition (standard, monkey and stimulus set (columns). The time $X$ frequency analysis window for a row is given above the row. $N$ denotes the number of analyzed stimulus $\times$ site combinations. Note that the scale along the $y$-axis varies across different panels.

surprise response (Lieder et al., 2013a). We did not see such a surprise response in our data when examining the difference between the responses to the deviant and reference as a function of the number of preceding stimuli within unaborted sequences (Fig. 8B).

A third possible cause could be the examined region, i.e., IT cortex. A recent MEG study (Susac et al., 2014), using gratings differing in spatial frequency, showed an occipital source of the vMMN. Source analyses of ERPs in human vMMN studies using complex stimuli suggest a contribution from the occipitotemporal cortex (Kimura et al., 2012; Li et al., 2012). Based on these observations, IT was a reasonable candidate to show a surprise response. However, it is possible that a surprise response in the oddball paradigm occurs in macaque parietal or prefrontal cortex. 
Boehnke et al. (2011) reported stronger sustained responses in monkey superior colliculus to both oddball flashed spots that were dimmer or brighter relative to a standard with an intermediate luminance. Although Boehnke et al. (2011) interpreted the increased responses to the deviants as "novelty responses," it is possible that the similar sustained responses for the dimmer and brighter stimuli are also present under equiprobable stimulation conditions and are not related to the stimuli being oddballs. Field potentials in the rabbit visual cortex (Astikainen et al., 2000), and the turtle tectum and forebrain (Prechtl and Bullock, 1993) showed no difference between the responses to deviants when presented in the oddball sequences or in isolation. Thus, the few existing intracranial recording studies that used a visual oddball paradigm and included an appropriate control agree with our findings in macaque IT.

The absence of a surprise response in monkey IT agrees with recent multiunit and LFP recordings in the primary auditory cortex of awake rats (Farley et al., 2010) and monkeys (Fishman and Steinschneider, 2012). Both studies, using the equiprobable control condition, found no surprise response to deviants. Ulanovsky et al. (2003) did observe stronger response in the anesthetized auditory cortex to a deviant compared with the same stimulus presented in an equiprobable control test. However, this putative surprise response was only observed for frequency differences between standard and deviant that were markedly larger than those present among the stimuli in the control test. As pointed out previously (Farley et al., 2010), the higher response to the deviant might have been due to a greater cross-stimulus adaptation in the equiprobable compared with the oddball sequence. The importance of cross-stimulus adaptation in the equiprobable control conditions has been demonstrated by Taaseh et al. (2011) and Hershenhoren et al. (2014) in single-unit recordings in the auditory cortex of anesthetized rats. This group also found that the responses to the deviant in the oddball sequences were underestimated by a model that incorporates cross-frequency adaptation, suggesting a real surprise response. However, such a conclusion is questionable because it critically depends on the validity of the model of stimulus-specific adaptation (May and Tiitinen, 2010; Mill et al., 2011). Because of the absence of a convincing surprise response in primary auditory cortex, it has been suggested that such a response underlying the auditory MMN is present in higher auditory areas. The latter is supported by a recent 7T fMRI study in humans (Szycik et al., 2013) but needs verification with intracranial recordings. This however might be different for the visual modality, because we did not find a surprise response in anterior IT.

Our findings may appear to contradict evidence for prediction-related responses in macaque IT by Meyer and Olson (2011) following statistical learning. However, Meyer and Olson (2011) studied IT responses after $>13 \mathrm{~d}$ of exposure to image pairs, which is much longer than the exposure in our study. It is likely, therefore, that the increased responses to the unexpected stimuli in the Meyer and Olson (2011) study reflect a long-term learning of two-stimulus sequences. Anderson and Sheinberg (2008) found a decreased instead of an increased response of IT neurons to stimuli that occurred at an unexpected time. Thus, unexpected events can also be associated with decreased IT responses.

The responses in IT in the visual oddball sequence are strongly determined by the most recently presented stimuli. This agrees with modeling work of somatosensory ERP data of a rovingstandard paradigm (Ostwald et al., 2012). Our IT data also show that stimuli further back in the sequence influence the response to the current stimulus, which disagrees with change detection models of responses in oddball sequences. Current models of responses in oddball paradigms state that the subject predicts the current stimulus based on the preceding sequence (Kimura, 2012; Ostwald et al., 2012; Lieder et al., 2013b). These models predict both a decrease in response to the standard and an increase in response to the deviant. With respect to the decreased response to the standard, note that a single stimulus presentation is sufficient to reduce the IT response to a subsequent presentation of that stimulus. In the context of the predictive coding model, this then implies that stimulus repetition is the default prediction (Kaliukhovich and Vogels, 2011). At least some predictive coding models predict that the response to a deviant increases as a function of the number of preceding standards (Ostwald et al., 2012), which we did not observe. Furthermore, predictive coding models assume that the responses in oddball sequences are strongly determined by top-down signals that carry the predictive signals. To our knowledge, there is no direct neurophysiological evidence showing top-down influences on adapted visual responses (e.g., following inactivation of higher areas). To the contrary, recent studies show that bottom-up models can explain adaptation effects for visual stimuli in extrastriate cortex (Patterson et al., 2014a,b). However, these studies were performed under anesthesia, which may have affected topdown modulations.

Our findings suggest that stimulus-specific adaptation underlies the responses in visual oddball paradigms in the higher visual cortical area IT. The response to the standard stimulus decreases nonlinearly with presentation number, reflecting stimulusspecific adaptation, without a surprise-related boost of activity to the deviant. Stimulus-specific adaptation can be viewed as an automatic mechanism whereby a stimulus that differs from recently seen ones will stand out in the context of the reduced response to the preceding stimuli (Wissig and Kohn, 2012). The present data show that for IT neurons these adaptive mechanisms take into account a relatively short stimulus history, with the effects at longer time scales being weak.

\section{References}

Anderson B, Sheinberg DL (2008) Effects of temporal context and temporal expectancy on neural activity in inferior temporal cortex. Neuropsychologia 46:947-957. CrossRef Medline

Antunes FM, Nelken I, Covey E, Malmierca MS (2010) Stimulus-specific adaptation in the auditory thalamus of the anesthetized rat. PLoS One 5:e14071. CrossRef Medline

Astikainen P, Hietanen JK (2009) Event-related potentials to task-irrelevant changes in facial expressions. Behav Brain Funct 5:30 CrossRef Medline

Astikainen P, Ruusuvirta T, Korhonen T (2000) Cortical and subcortical visual event-related potentials to oddball stimuli in rabbits. Neuroreport 11:1515-1517. CrossRef Medline

Barto A, Mirolli M, Baldassarre G (2013) Novelty or surprise? Front Psychol 4:907. CrossRef Medline

Baylis GC, Rolls ET (1987) Responses of neurons in the inferior temporal cortex in short term and serial recognition memory tasks. Exp Brain Res 65:614-622. Medline

Boehnke SE, Berg DJ, Marino RA, Baldi PF, Itti L, Munoz DP (2011) Visual adaptation and novelty responses in the superior colliculus. Eur J Neurosci 34:766-779. CrossRef Medline

Buzsáki G, Anastassiou CA, Koch C (2012) The origin of extracellular fields and currents: EEG, ECoG, LFP and spikes. Nat Rev Neurosci 13:407-420. CrossRef Medline

Costa-Faidella J, Grimm S, Slabu L, Díaz-Santaella F, Escera C (2011) Multiple time scales of adaptation in the auditory system as revealed by human evoked potentials. Psychophysiology 48:774-783. CrossRef Medline

Cowan N, Winkler I, Teder W, Näätänen R (1993) Memory prerequisites of mismatch negativity in the auditory event-related potential (ERP). J Exp Psychol Learn Mem Cogn 19:909-921. CrossRef Medline 
Czigler I (2007) Visual mismatch negativity: violation of nonattended environmental regularities. J Psychophysiol 21:224-230. CrossRef

Czigler I, Balázs L, Winkler I (2002) Memory-based detection of taskirrelevant visual changes. Psychophysiology 39:869-873. CrossRef Medline

De Baene W, Vogels R (2010) Effects of adaptation on the stimulus selectivity of macaque inferior temporal spiking activity and local field potentials. Cereb Cortex 20:2145-2165. CrossRef Medline

DiCarlo JJ, Zoccolan D, Rust NC (2012) How does the brain solve visual object recognition? Neuron 73:415-434. CrossRef Medline

Duncan CC, Barry RJ, Connolly JF, Fischer C, Michie PT, Näätänen R, Polich J, Reinvang I, Van Petten C (2009) Event-related potentials in clinical research: guidelines for eliciting, recording, and quantifying mismatch negativity, P300, and N400. Clin Neurophysiol 120:1883-1908. CrossRef Medline

Efron B (1979) Bootstrap methods: another look at the jackknife. Ann Statist 7:1-26. CrossRef

Einevoll GT, Kayser C, Logothetis NK, Panzeri S (2013) Modelling and analysis of local field potentials for studying the function of cortical circuits. Nat Rev Neurosci 14:770-785. CrossRef Medline

Engbert R, Kliegl R (2003) Microsaccades uncover the orientation of covert attention. Vis Res 43:1035-1045. CrossRef Medline

Farley BJ, Quirk MC, Doherty JJ, Christian EP (2010) Stimulus-specific adaptation in auditory cortex is an NMDA-independent process distinct from the sensory novelty encoded by the mismatch negativity. J Neurosci 30:16475-16484. CrossRef Medline

Fishman YI, Steinschneider M (2012) Searching for the mismatch negativity in primary auditory cortex of the awake monkey: deviance detection or stimulus specific adaptation? J Neurosci 32:15747-15758. CrossRef Medline

Garrido MI, Friston KJ, Kiebel SJ, Stephan KE, Baldeweg T, Kilner JM (2008) The functional anatomy of the MMN: a DCM study of the roving paradigm. Neuroimage 42:936-944. CrossRef Medline

Garrido MI, Kilner JM, Stephan KE, Friston KJ (2009) The mismatch negativity: a review of underlying mechanisms. Clin Neurophysiol 120:453463. CrossRef Medline

Gross CG, Schiller PH, Wells C, Gerstein GL (1967) Single-unit activity in temporal association cortex of the monkey. J Neurophysiol 30:833-843. Medline

Gross CG, Bender DB, Rocha-Miranda CE (1969) Visual receptive fields of neurons in inferotemporal cortex of the monkey. Science 166:1303-1306. CrossRef Medline

Hershenhoren I, Taaseh N, Antunes FM, Nelken I (2014) Intracellular correlates of stimulus-specific adaptation. J Neurosci 34:3303-3319. CrossRef Medline

Jacobsen T, Schröger E (2001) Is there pre-attentive memory-based comparison of pitch? Psychophysiology 38:723-727. CrossRef Medline

Kaliukhovich DA, Vogels R (2011) Stimulus repetition probability does not affect repetition suppression in macaque inferior temporal cortex. Cereb Cortex 21:1547-1558. CrossRef Medline

Kaliukhovich DA, Vogels R (2012) Stimulus repetition affects both strength and synchrony of macaque inferior temporal cortical activity. J Neurophysiol 107:3509-3527. CrossRef Medline

Kecskés-Kovács K, Sulykos I, Czigler I (2013a) Visual mismatch negativity is sensitive to symmetry as a perceptual category. Eur J Neurosci 37:662667. CrossRef Medline

Kecskés-Kovács K, Sulykos I, Czigler I (2013b) Is it a face of a woman or a man? Visual mismatch negativity is sensitive to gender category. Front Hum Neurosci 7:532. CrossRef Medline

Kekoni J, Hämäläinen H, Saarinen M, Gröhn J, Reinikainen K, Lehtokoski A, Näätänen R (1997) Rate effect and mismatch responses in the somatosensory system: ERP recordings in humans. Biol Psychol 46:125-142. CrossRef Medline

Kimura M (2012) Visual mismatch negativity and unintentional temporalcontext-based prediction in vision. Int J Psychophysiol 83:144-155. CrossRef Medline

Kimura M, Katayama J, Murohashi H (2006) Probability-independent and -dependent ERPs reflecting visual change detection. Psychophysiology 43:180-189. CrossRef Medline

Kimura M, Kondo H, Ohira H, Schröger E (2012) Unintentional temporal context-based prediction of emotional faces: an electrophysiological study. Cereb Cortex 22:1774-1785. CrossRef Medline
Li X, Lu Y, Sun G, Gao L, Zhao L (2012) Visual mismatch negativity elicited by facial expressions: new evidence from the equiprobable paradigm. Behav Brain Funct 8:7. CrossRef Medline

Lieder F, Daunizeau J, Garrido MI, Friston KJ, Stephan KE (2013a) Modelling trial-by-trial changes in the mismatch negativity. PLoS Comput Biol 9:e1002911. CrossRef Medline

Lieder F, Stephan KE, Daunizeau J, Garrido MI, Friston KJ (2013b) A neurocomputational model of the mismatch negativity. PLoS Comput Biol 9:e1003288. CrossRef Medline

Liu Y, Murray SO, Jagadeesh B (2009) Time course and stimulus dependence of repetition-induced response suppression in inferotemporal cortex. J Neurophysiol 101:418-436. CrossRef Medline

Logothetis NK (2003) The underpinnings of the BOLD functional magnetic resonance imaging signal. J Neurosci 23:3963-3971. Medline

Logothetis NK, Sheinberg DL (1996) Visual object recognition. Annu Rev Neurosci 19:577-621. CrossRef Medline

Maekawa T, Tobimatsu S, Ogata K, Onitsuka T, Kanba S (2009) Preattentive visual change detection as reflected by the mismatch negativity (MMN): evidence for a memory-based process. Neurosci Res 65:107-112. CrossRef Medline

May PJ, Tiitinen H (2010) Mismatch negativity (MMN), the devianceelicited auditory deflection, explained. Psychophysiology 47:66-122. CrossRef Medline

Meyer T, Olson CR (2011) Statistical learning of visual transitions in monkey inferotemporal cortex. Proc Natl Acad Sci U S A 108:19401-19406. CrossRef Medline

Mill R, Coath M, Wennekers T, Denham SL (2011) A neurocomputational model of stimulus-specific adaptation to oddball and Markov sequences. PLoS Comput Biol 7:e1002117. CrossRef Medline

Miller EK, Gochin PM, Gross CG (1991a) Habituation-like decrease in the responses of neurons in inferior temporal cortex of the macaque. Vis Neurosci 7:357-362. CrossRef Medline

Miller EK, Li L, Desimone R (1991b) A neural mechanism for working and recognition memory in inferior temporal cortex. Science 254:1377-1379. CrossRef Medline

Mitzdorf U (1987) Properties of the evoked potential generators: current source-density analysis of visually evoked potentials in the cat cortex. Int J Neurosci 33:33-59. CrossRef Medline

Näätänen R, Gaillard AWK, Mäntysalo S (1978) Early selective-attention effect on evoked potential reinterpreted. Acta Psychol 42:313-329. CrossRef

Näätänen R, Jacobsen T, Winkler I (2005) Memory-based or afferent processes in mismatch negativity (MMN): a review of the evidence. Psychophysiology 42:25-32. CrossRef Medline

Noudoost B, Esteky H (2013) Neuronal correlates of view representation revealed by face-view aftereffect. J Neurosci 33:5761-5772. CrossRef Medline

Ostwald D, Spitzer B, Guggenmos M, Schmidt TT, Kiebel SJ, Blankenburg F (2012) Evidence for neural encoding of Bayesian surprise in human somatosensation. Neuroimage 62:177-188. CrossRef Medline

Patterson CA, Wissig SC, Kohn A (2014a) Adaptation disrupts motion integration in the primate dorsal stream. Neuron 81:674-686. CrossRef Medline

Patterson CA, Duijnhouwer J, Wissig SC, Krekelberg B, Kohn A (2014b) Similar adaptation effects in primary visual cortex and area MT of the macaque monkey under matched stimulus conditions. J Neurophysiol 111:1203-1213. CrossRef Medline

Popivanov ID, Jastorff J, Vanduffel W, Vogels R (2012) Stimulus representations in body-selective regions of the macaque cortex assessed with event-related fMRI. Neuroimage 63:723-741. CrossRef Medline

Prechtl JC, Bullock TH (1993) Plurality of visual mismatch potentials in a reptile. J Cogn Neurosci 5:177-187. CrossRef Medline

Sawamura H, Orban GA, Vogels R (2006) Selectivity of neuronal adaptation does not match response selectivity: a single-cell study of the fMRI adaptation. Neuron 49:307-318. CrossRef Medline

Schröger E, Wolff C (1996) Mismatch response of the human brain to changes in sound location. Neuroreport 7:3005-3008. CrossRef Medline

Stefanics G, Csukly G, Komlósi S, Czobor P, Czigler I (2012) Processing of unattended facial emotions: a visual mismatch negativity study. Neuroimage 59:3042-3049. CrossRef Medline

Sulykos I, Kecskés-Kovács K, Czigler I (2013) Mismatch negativity does not 
show evidence of memory reactivation in the visual modality. J Psychophysiol 27:1-6. CrossRef

Susac A, Heslenfeld DJ, Huonker R, Supek S (2014) Magnetic source localization of early visual mismatch response. Brain Topogr 27:648-651. CrossRef Medline

Szycik GR, Stadler J, Brechmann A, Münte TF (2013) Preattentive mechanisms of change detection in early auditory cortex: a 7 tesla fMRI study. Neuroscience 253:100-109. CrossRef Medline

Taaseh N, Yaron A, Nelken I (2011) Stimulus-specific adaptation and deviance detection in the rat auditory cortex. PLoS One 6:e23369. CrossRef Medline

Tallon-Baudry C, Bertrand O (1999) Oscillatory gamma activity in humans and its role in object representation. Trends Cogn Sci 3:151-162. CrossRef Medline

Tanaka K (1996) Inferotemporal cortex and object vision. Annu Rev Neurosci 19:109-139. CrossRef Medline
Tsao DY, Livingstone MS (2008) Mechanisms of face perception. Annu Rev Neurosci 31:411-437. CrossRef Medline

Ulanovsky N, Las L, Nelken I (2003) Processing of low-probability sounds by cortical neurons. Nat Neurosci 6:391-398. CrossRef Medline

Ulanovsky N, Las L, Farkas D, Nelken I (2004) Multiple time scales of adaptation in auditory cortex neurons. J Neurosci 24:10440-10453. CrossRef Medline

Winkler I, Karmos G, Näätänen R (1996) Adaptive modeling of the unattended acoustic environment reflected in the mismatch negativity eventrelated potential. Brain Res 742:239-252. CrossRef Medline

Wissig SC, Kohn A (2012) The influence of surround suppression on adaptation effects in primary visual cortex. J Neurophysiol 107:3370-3384. CrossRef Medline

Zhao L, Liu Y, Shen L, Feng L, Hong B (2011) Stimulus-specific adaptation and its dynamics in the inferior colliculus of rat. Neuroscience 181:163174. CrossRef Medline 